\title{
Scaling laws in X-ray galaxy clusters at redshift between 0.4 and $1.3^{\star}$
}

\author{
S. Ettori ${ }^{1}$, P. Tozzi ${ }^{2}$, S. Borgani ${ }^{3,4}$, and P. Rosati ${ }^{1}$ \\ 1 ESO, Karl-Schwarzschild-Str. 2, 85748 Garching, Germany \\ 2 INAF, Osservatorio Astronomico di Trieste, via G. B. Tiepolo 11, 34131 Trieste, Italy \\ 3 Dip. di Astronomia, Universitá di Trieste, via G. B. Tiepolo 11, 34131 Trieste, Italy \\ 4 INFN - Istituto Nazionale di Fisica Nucleare, Trieste, Italy
}

Received 28 July 2003 / Accepted 29 November 2003

\begin{abstract}
We present a study of the integrated physical properties of a sample of $28 \mathrm{X}$-ray galaxy clusters observed with Chandra at a redshift between 0.4 and 1.3. In particular, we have twelve objects in the redshift range 0.4-0.6, five between 0.6 and 0.8 , seven between 0.8 and 1 and four at $z>1.0$, compounding the largest sample available for such a study. We focus particularly on the properties and evolution of the X-ray scaling laws. We fit both a single and a double $\beta$-model with the former which provides a good representation of the observed surface brightness profiles, indicating that these clusters do not show any significant excess in their central brightness. By using the best-fit parameters of the $\beta$-model together with the measured emission-weighted temperature (in the range 3-11 keV), we recover gas luminosity, gas mass and total gravitating mass out to $R_{500}$. We observe scaling relations steeper than expected from the self-similar model by a significant $(>3 \sigma)$ amount in the $L-T$ and $M_{\mathrm{gas}}-T$ relations and by a marginal value in the $M_{\mathrm{tot}}-T$ and $L-M_{\mathrm{tot}}$ relations. The degree of evolution of the $M_{\mathrm{tot}}-T$ relation is found to be consistent with the expectation based on the hydrostatic equilibrium for gas within virialized dark matter halos. We detect hints of negative evolution in the $L-T, M_{\mathrm{gas}}-T$ and $L-M_{\mathrm{tot}}$ relations, thus suggesting that systems at higher redshift have lower X-ray luminosity and gas mass for fixed temperature. In particular, when the 16 clusters at $z>0.6$ are considered, the evolution becomes more evident and its power-law modelization is a statistically good description of the data. In this subsample, we also find significant evidence for positive evolution, such as $(1+z)^{0.3}$, in the $E_{z}^{4 / 3} S-T$ relation, where the entropy $S$ is defined as $T / n_{\text {gas }}^{2 / 3}$ and is measured at $0.1 R_{200}$. Such results point toward a scenario in which a relatively lower gas density is present in high-redshift objects, thus implying a suppressed X-ray emission, a smaller amount of gas mass and a higher entropy level. This represents a non-trivial constraint for models aiming at explaining the thermal history of the intra-cluster medium out to the highest redshift reached so far.
\end{abstract}

Key words. galaxies: cluster: general - galaxies: fundamental parameters - galaxies: intergalactic medium - X-ray: galaxies cosmology: observations - cosmology: dark matter

\section{Introduction}

The physics of the intracluster medium (ICM) is mainly driven by the infall of the cosmic baryons trapped in the deep gravitational potential of the cluster dark matter halo. Through a hierarchical formation that starts from the primordial density fluctuations and generates the largest virialized structures via gravitational collapse and merging, galaxy clusters maintain similar properties when they are rescaled with respect to gravitational mass and epoch of formation. The shock-heated X-ray emitting ICM accounts for most of the baryons collapsed in the cluster potential (e.g. White et al. 1993). Its physical properties, like density and temperature, relate in a predictable way in this simple self-similar scenario (e.g. Kaiser 1986, 1991; Evrard \& Henry 1991). Under the assumptions that the smooth and spherically symmetric ICM is heated only by gravitational

\footnotetext{
Send offprint requests to: S. Ettori, e-mail: settori@eso.org

* Figure A1 is only available in electronic form at http://www. edpsciences.org
}

processes (adiabatic compression during DM collapse and shock heating from supersonic accretion), obeys hydrostatic equilibrium within the underlying dark matter potential and emits mainly by bremsstrahlung, one derives the following scaling relations between the observed (bolometric luminosity $L_{\text {bol }}$, and temperature $T_{\text {gas }}$ ) and derived (gas entropy $S$, gas mass $M_{\mathrm{gas}}$, and total gravitating mass $M_{\mathrm{tot}}$ ) quantities:

- $E_{z}^{4 / 3} S \propto \Delta_{z}^{-2 / 3} T_{\text {gas }}$

- $E_{z}^{-1} L_{\mathrm{bol}} \propto \Delta_{z}^{1 / 2} T_{\mathrm{gas}}^{2}$

- $E_{z} M_{\text {tot }} \propto \Delta_{z}^{-1 / 2} T_{\text {gas }}^{3 / 2}$

- $E_{z}^{-1} L_{\mathrm{bol}} \propto \Delta_{z}^{7 / 6}\left(E_{z} M_{\mathrm{tot}}\right)^{4 / 3}$

- $E_{z} M_{\mathrm{gas}} \propto \Delta_{z}^{-1 / 2} T_{\mathrm{gas}}^{3 / 2}$.

The factors that indicate the dependence on the evolution of the Hubble constant at redshift $z, E_{z}=H_{z} / H_{0}=$ $\left[\Omega_{\mathrm{m}}(1+z)^{3}+1-\Omega_{\mathrm{m}}\right]^{1 / 2}$ (for a flat cosmology with matter density $\Omega_{\mathrm{m}}$ ), and on the overdensity $\Delta_{z}$ account for the fact that all the quantities are estimated at a given overdensity $\Delta_{z}$ 
with respect to the critical density estimated at redshift $z$, $\rho_{\mathrm{c}, \mathrm{z}}=3 H_{z}^{2} /(8 \pi G)$.

Hydrodynamical simulations (e.g. Evrard et al. 1996; Bryan \& Norman 1998; Thomas et al. 2001; Bialek et al. 2001; Borgani et al. 2002 and references therein) and observational analyses (e.g. from Mushotzky 1984; Edge \& Stewart 1991 to the more recent work of Allen \& Fabian 1998; Markevitch 1998; Arnaud \& Evrard 1999; Nevalainen et al. 2000; Finoguenov et al. 2001; Ettori et al. 2002) of the beststudied correlations between X-ray luminosity, total gravitating mass and gas temperature show that significant deviations exist between observations and the expectations based on selfsimilar scaling: (i) a difference of the order of 30-40 per cent in the normalization of the $M_{\text {tot }}-T_{\text {gas }}$ relation (Horner et al. 1999; Nevalainen et al. 2000); (ii) steeper slopes of the $L_{\mathrm{bol}}-$ $T, M_{\mathrm{gas}}-T$ and, possibly, $M_{\mathrm{tot}}-T$ relations, in particular when low temperature $\left(T_{\text {gas }}<3 \mathrm{keV}\right)$ systems are also considered; (iii) excess entropy in the central regions of clusters and groups, with respect to the $S \propto T$ expectation (e.g., Ponman et al. 1999, 2003). Such deviations are currently interpreted as evidence for non-gravitational processes, such as extra heating and radiative cooling, that affect the assembly of the baryons in the cluster potential well (e.g. Cavaliere et al. 1999; Bower et al. 2001; Tozzi \& Norman 2001; Babul et al. 2002; Voit et al. 2002).

The same processes that determine the shape of the local relations should have affected also the evolution of the scaling laws with the cosmic epoch. However, due to the objective difficulty to assemble a large dataset of high redshift objects, there has been very little work done until now, and with contradictory results, on the observed evolution of the scaling laws. For example, for different subsamples of our dataset, Holden et al. (2002) observe no evolution of the $L_{\text {bol }}-T_{\text {gas }}$ relation in a $\Lambda$ CDM universe, while Vikhlinin et al. (2002) claim to have evidence at $8 \sigma$ level for a positive evolution, i.e. systems at higher redshift have higher luminosity at fixed temperature. In principle, any observed evolution in the scaling laws allows to constrain different scenarios of heating schemes and cooling efficiency.

Furthermore, understanding the evolution of scaling relations involving luminosity, temperature and total collapsed mass, is of vital importance if the evolution of the cluster population has to be used as a proxy to the cosmic evolution and, therefore, for the determination of cosmological parameters (e.g., the pioneering work of Henry \& Arnaud 1991; see Rosati et al. 2002, for a recent review and references therein). In fact, while the mass function of galaxy clusters and its evolution are sensitive probes of the underlying cosmological scenario, mass is usually inferred from observational quantities, such as the $\mathrm{X}$-ray luminosity and the gas temperature, which are affected by non-gravitational processes.

In this paper, we focus on the general behaviour of the scaling laws for X-ray clusters at high redshift, with the main aim of quantifying their evolution with respect to what is observed for nearby clusters. To do this, we have assembled a set of 28 clusters in the redshift range $0.4-1.3$ observed at arcsec angular resolution with Chandra (Weisskopf et al. 2000). This allows us to resolve on scales of few tens of kpc the surface brightness (i.e. the gas density) of these systems, and to determine a single emission weighted temperature. In the next section, we describe our dataset and how the physical quantities are measured from the observations. We present in Sect. 3 the results on the scaling laws under examination and in Sect. 4 the constraints on the observed evolution of these relations. We summarize our conclusions in Sect. 5. All quoted errors are at $1 \sigma$ level (68.3 per cent level of confidence and $\Delta \chi^{2}=1$ for one interesting parameter) unless otherwise stated. The cosmological parameters $H_{0}=70 h_{70}^{-1} \mathrm{~km} \mathrm{~s}^{-1} \mathrm{Mpc}^{-1}$ and $\Omega_{\mathrm{m}}=1-\Omega_{\Lambda}=0.3$ are assumed hereafter as suggested from WMAP constraints on the anisotropies in the cosmic microwave background (Spergel et al. 2003).

\section{The dataset}

As of Summer 2003, we consider all the clusters available in the Chandra archive with redshift larger than 0.4. These 28 galaxy clusters have redshift between 0.4 and 1.3 (median $z=0.73$ ), emission-weighted temperature in the range $3-11 \mathrm{keV}$ (median value of $6.0 \mathrm{keV}$ ) and luminosity between $10^{44} \mathrm{erg} \mathrm{s}^{-1}$ and $1.2 \times 10^{46} \mathrm{erg} \mathrm{s}^{-1}$ (median value: $6.9 \times$ $\left.10^{44} \mathrm{erg} \mathrm{s}^{-1}\right)$. In particular, twelve of these objects were selected from the ROSAT Deep Cluster Survey (RDCS; Rosati et al. 1998; Stanford et al. 2001; Holden et al. 2002; Rosati et al. 2003) of which we present the complete Chandra followup of objects with $z>0.8$, seven from the Einstein Extended Medium Sensitivity Survey (MS; Gioia et al. 1990), four from the 160 Square Degrees ROSAT Survey (Vikhlinin et al. 1998; Mullis et al. 2003), RX J1113 and WGA1226 from the Wide Angle ROSAT Pointed Survey (WARPS; Perlman et al. 2002; Maughan et al. 2003; see also Cagnoni et al. 2001), RX J1716 is part of the North Ecliptic Pole survey (NEP; Gioia et al. 1999; Henry et al. 2001), RX J1347 has been discovered in the ROSAT All Sky Survey (Schindler et al. 1995) and 3C 295 has been already mapped with Einstein (Henry \& Henriksen 1986). Subsamples of the present one have been used to constrain the cosmological parameters through the cluster baryonic content (Ettori et al. 2003) and the iron abundance in the ICM at high redshift (Tozzi et al. 2003).

The data reduction is described in details in Tozzi et al. (2003). We summarize here its most relevant aspects. We reprocess the level $=1$ events files retrieved from the archive. When the observations were in VFAINT mode, the pulse height in a $5 \times 5$ event island has been used. The corrections for Charge Transfer Inefficiency (CTI) have been applied when the Focal Plane temperature was 153 K. Finally, we apply a double correction to the ARF files. We apply the script apply_acisabs by Chartas and Getman to take into account the degradation in the ACIS QE due to material accumulated on the ACIS optical blocking filter since launch (see http://cxc.harvard.edu/ciao/ threads/applyacisabs/ and Marshall et al. 2003). This correction apply to all the observation. For data taken with ACIS-I (note that only RX J1347, 3C 295, MS0451, MS1054 and WGA1226 have been observed with the back-illuminated S3 CCD), we also apply manually a correction to the effective area consisting in a $7 \%$ decrement below $1.8 \mathrm{keV}$ (see Markevitch \& Vikhlinin 2001). 


\subsection{X-ray analysis}

The spectra are extracted out to a maximum radius $R_{\text {spec }}$, which is chosen cluster by cluster so as to optimize the signal-to-noise ratio in the $0.5-5 \mathrm{keV}$ band where most of the Chandra effective area is available. They are modeled between 0.6 and $8 \mathrm{keV}$ with an absorbed optically-thin plasma (tbabs (mekal) in XSPEC v. 11.2, Arnaud 1996) with redshift fixed to the value available in literature and absorption equal to the Galactic value (as recovered from radio HI maps, Dickey \& Lockman 1990) in correspondence of the X-ray peak. A local background obtained from regions of the same CCD free of any point source is used. The gas temperature, $T_{\text {gas }}$, metallicity and the normalization $K$ of the thermal component are the only free parameters of the spectral fit performed with a Cash statistics due to the low number of counts per bin available (e.g. Nousek \& Shue 1989; see also discussion on background-subtracted spectra at http://heasarc.gsfc.nasa.gov/docs/xanadu/xspec/ manual/node57.html).

The surface brightness profiles are obtained from the exposure-corrected image by fixing the number of counts per bin between 50 and 200, depending on the photons available, and is then fitted with an isothermal $\beta$-model (Cavaliere \& Fusco-Femiano 1976). This model provides a reasonably good fit to all the profiles (see plots in Appendix), apart from seven clusters whose measured profiles have a probability $<0.01$ not to be a random realization of a $\beta$-model, as drawn from a $\chi^{2}$ distribution (RX J1416, $\chi_{\text {red }}^{2}=1.98 ; \mathrm{RX} \mathrm{J1701,} \chi_{\text {red }}^{2}=1.96$; $\mathrm{RX} \mathrm{J1525,} \chi_{\text {red }}^{2}=1.47 ;$ MS0451, $\chi_{\text {red }}^{2}=1.45 ;$ MS0016, $\chi_{\text {red }}^{2}=$ $\left.1.40 ; \mathrm{RX} \mathrm{J1113,} \chi_{\text {red }}^{2}=1.49 ; \mathrm{RX} \mathrm{J} 2302, \chi_{\text {red }}^{2}=1.59\right)$. This is not unexpected, being now well known that highly spatially resolved observations of nearby systems have surface brightness profiles poorly described by simple functional forms, due to the presence of breaks and general irregularities that cannot be reproduced by any smoothed curve (see, e.g., the case of A2390 in Allen et al. 2001b and the detected front in RX J1252, Rosati et al. 2003) and overall two-dimensional shape not strictly circular as here assumed (e.g. Maughan et al. 2003 on RX J1113 and Donahue et al. 2003 on MS0451). On the other hand, the results obtained from the application of the $\beta$-model represent a robust estimates of the effective values under exam, such as gas and total gravitating mass, as shown for simulated clusters (e.g. Mohr et al. 1999).

We have also tested a double $\beta$-model in the form suggested by Ettori (2000 and reference therein), which provides a consistent physical framework for the modeling: $S_{\mathrm{b}}=S_{0, \text { inn }}\left(1-x^{2}\right)^{0.5+3 \beta_{\text {inn }}}+S_{0}\left(1+x^{2}\right)^{0.5-3 \beta}=$ $r_{\mathrm{c}} \Lambda(T) n_{0, \text { gas }}^{2}\left[f_{\text {inn }} B\left(0.5,3 \beta_{\text {inn }}+1\right)\left(1-x^{2}\right)^{0.5+3 \beta_{\text {inn }}}+\left(1-f_{\text {inn }}\right) B(3 \beta-\right.$ $\left.0.5,0.5)\left(1+x^{2}\right)^{0.5-3 \beta}\right]$, where $r_{\mathrm{c}}$ is the common core radius, $x=r / r_{\mathrm{c}}, \Lambda(T)$ is the cooling function, $n_{0, \mathrm{gas}}$ is the central gas density and $B()$ is the incomplete beta function. From a statistical point of view, none of the clusters requires to add two parameters (i.e. the slope $\beta_{\text {inn }}$ of the inner, corrected $\beta$-model and the relative contribution $f_{\text {inn }}$ of the central, cool component). The F-test (Bevington \& Robinson 1992, p.205ff) gives probability values in the range between 0.40 (MS1054) and 0.85 (MS0016), so that we can conclude that a double $\beta$-model is not required by our data at the fixed threshold of 0.95 (95 per cent confidence level). In particular, we note that RX J1416 and RX J1701, the two clusters with worst $\chi^{2}$ associated to the single $\beta$-model, show the smaller core radii, but still cannot accommodate a double $\beta$-model that provides a F-test statistic with probability of 0.82 and 0.67 , respectively. On the other hand, few clusters at redshift around 0.3 not included in the present sample, such as MS1008 and MS2137, require a double $\beta$-model with probability of $>0.99$ and 0.98 , respectively. It is worth noticing two items concerning the surface brightness of high $-z$ clusters. First, thanks to the Chandra spatial resolution, we are in the condition to resolve on $\sim 20 \mathrm{kpc}$ scale their inner regions. For example, we are able to define between 4 and 7 spatial bins with a fixed number of 50 counts within $100 \mathrm{kpc}$ in the four $z>1$ objects. Then, the evidence that their surface brightness cannot accommodate a double $\beta$-model indicates that these clusters do not show any significant excess in their central brightness, consistent with the picture that systems at high redshift appear as structures in formation and not completely relaxed. We refer to a forthcoming paper (Ettori et al. in prep.) for a more detailed analysis of the distribution of the observed surface brightness, in particular in the central regions, as function of the object's redshift.

We have investigated the presence of trends between the best-fit parameters of the $\beta$-model, the redshift and the gas temperature of the objects in the overall sample by evaluating the significance of the Spearman's rank correlation. We obtain a relevant deviation (Spearman's rank correlation with a probability $P=4 \times 10^{-5}$ ) from the no-correlation case only for the well-known degeneracy between $r_{\mathrm{c}}$ and $\beta$ values, being higher $\beta$ measured in correspondence of larger core radii (see Fig. 1; for the same correlation observed in nearby and intermediate-redshift objects see, e.g., Ettori \& Fabian 1999). A mild trend $(P=0.03)$ between $r_{\mathrm{c}}$ and $T_{\text {gas }}$ is also observed. The latter correlation has a milder correspondence between $\beta$ and $T_{\text {gas }}(P=0.17)$ and indicates that larger core radii are measured in more massive systems, with no sizable change in the outer slope of the density profile. As shown in Fig. 1 and quantified in Table 2, the best-fit parameters $r_{\mathrm{c}}$ and $\beta$ do not show any significant trend with the redshift.

The advantage of using a $\beta$-model to parameterize the observed surface brightness is that gas density and total mass profiles can be recovered analytically and expressed by simple formula:

$$
\begin{aligned}
& n_{\mathrm{gas}}(r)=n_{0, \mathrm{gas}}\left(1+x^{2}\right)^{-3 \beta / 2} \\
& M_{\mathrm{tot}}(<r)=\frac{3 \beta T_{\mathrm{gas}} r_{\mathrm{c}}}{G \mu m_{\mathrm{p}}} \frac{x^{3}}{1+x^{2}},
\end{aligned}
$$

where $x=r / r_{\mathrm{c}}, \mu$ is the mean molecular weight in atomic mass unit (=0.6), $G$ is the gravitational constant and $m_{\mathrm{p}}$ is the proton mass.

In particular, the central electron density is obtained from the combination of the best-fit results from the spectral and imaging analyses as follows:

$n_{0, \text { ele }}^{2}=\frac{4 \pi d_{\mathrm{ang}}^{2} \times(1+z)^{2} \times K \times 10^{14}}{0.82 \times 4 \pi r_{\mathrm{c}}^{3} \times E I}$ 


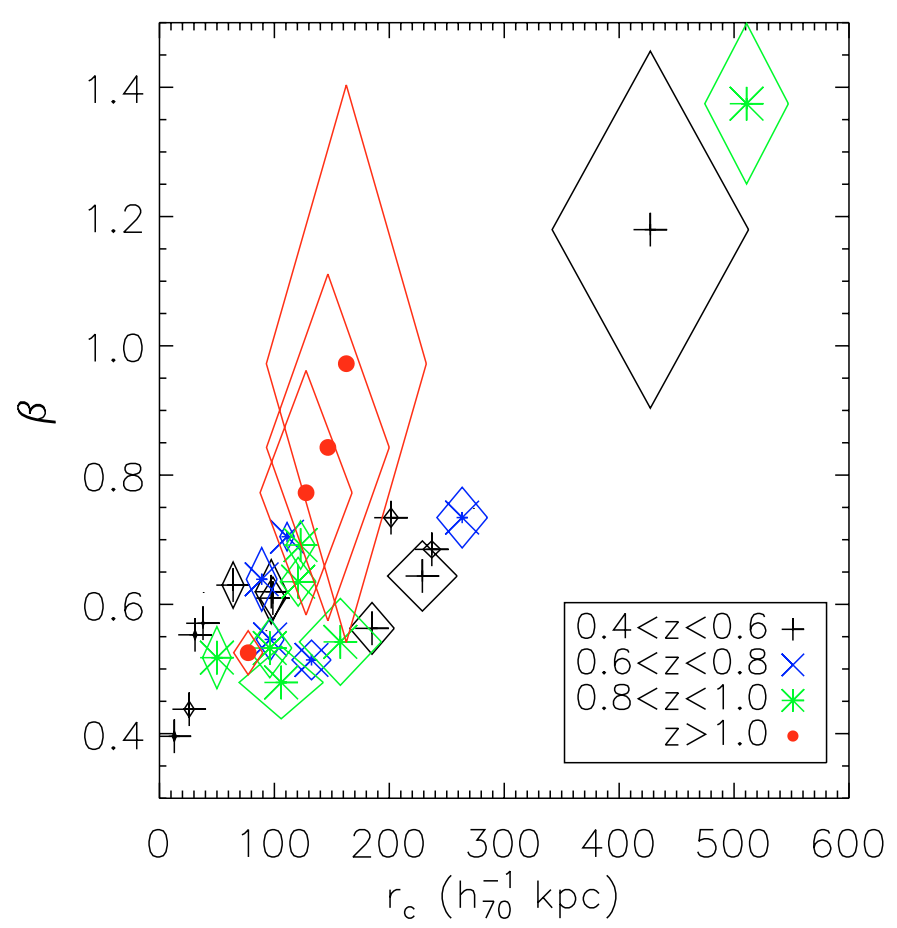

Fig. 1. Correlations between the best-fit parameters of the $\beta$-model: higher values of the core radius, $r_{\mathrm{c}}$, correspond to higher estimates of $\beta$. No redshift-segregation is evident.

where $d_{\text {ang }}$ is the angular-size distance, $K=10^{-14} \times$ $\int n_{\mathrm{p}} n_{\mathrm{e}} \mathrm{d} V /\left[4 \pi d_{\text {ang }}^{2}(1+z)^{2}\right]$ is the normalization of the thermal spectrum measured within XSPEC and the emission integral $E I$ is estimated by integrating the emission from the spherical source out to $10 \mathrm{Mpc}$ along the line of sight, $E I=$ $\int_{0}^{x_{1}}\left(1+x^{2}\right)^{-3 \beta} x^{2} \mathrm{~d} x+\int_{x_{1}}^{x_{2}}\left(1+x^{2}\right)^{-3 \beta} x^{2}(1-\cos \theta) \mathrm{d} x$, with $\theta=$ $\arcsin \left(R_{\text {spec }} / r\right), x_{1}=R_{\text {spec }} / r_{\mathrm{c}}$ and $x_{2}=10 \mathrm{Mpc} / r_{\mathrm{c}},\left(\beta, r_{\mathrm{c}}\right)$ are the best-fit parameters of the $\beta$-model and we assume $n_{\mathrm{p}}=0.82 n_{\mathrm{e}}$ in the ionized intra-cluster plasma. To evaluate the relative errors on the estimated quantities, we repeat the measurements after 1000 random selection of a temperature, normalization $K$ and surface brightness profile, which were drawn from Gaussian distributions with mean and variance in accordance to the best-fit results. By this process, we obtain a distribution of the estimates of gas density, gas mass and total mass. The median value and the 16th and 84th percentile of each distribution are adopted as central and $(-1,1) \sigma$ interval, respectively (see Table 1).

From the total mass profile, $M_{\text {tot }}(<r)$, obtained for each galaxy cluster observed at redshift $z$, we then evaluate $R_{\Delta}$ as the radius encompassing a fixed density contrast, $\Delta_{z}$, with respect to the critical density, $\rho_{\mathrm{c}, \mathrm{z}}$, as

$\Delta_{z}=\frac{3 M_{\mathrm{tot}}\left(<R_{\Delta}\right)}{4 \pi \rho_{\mathrm{c}, \mathrm{z}} R_{\Delta}^{3}}$.

Observational results for nearby clusters (e.g. De Grandi \& Molendi 2002; Pratt \& Arnaud 2002) show that temperature profiles do not decline significantly ( $\$ 20$ per cent) out to an overdensity $\sim 500$ (for a universe with $\Omega_{\mathrm{m}}=1$ and $\Omega_{\Lambda}=0$ ). Therefore, to make a proper use of our single-temperature measurement, we estimate all the observed

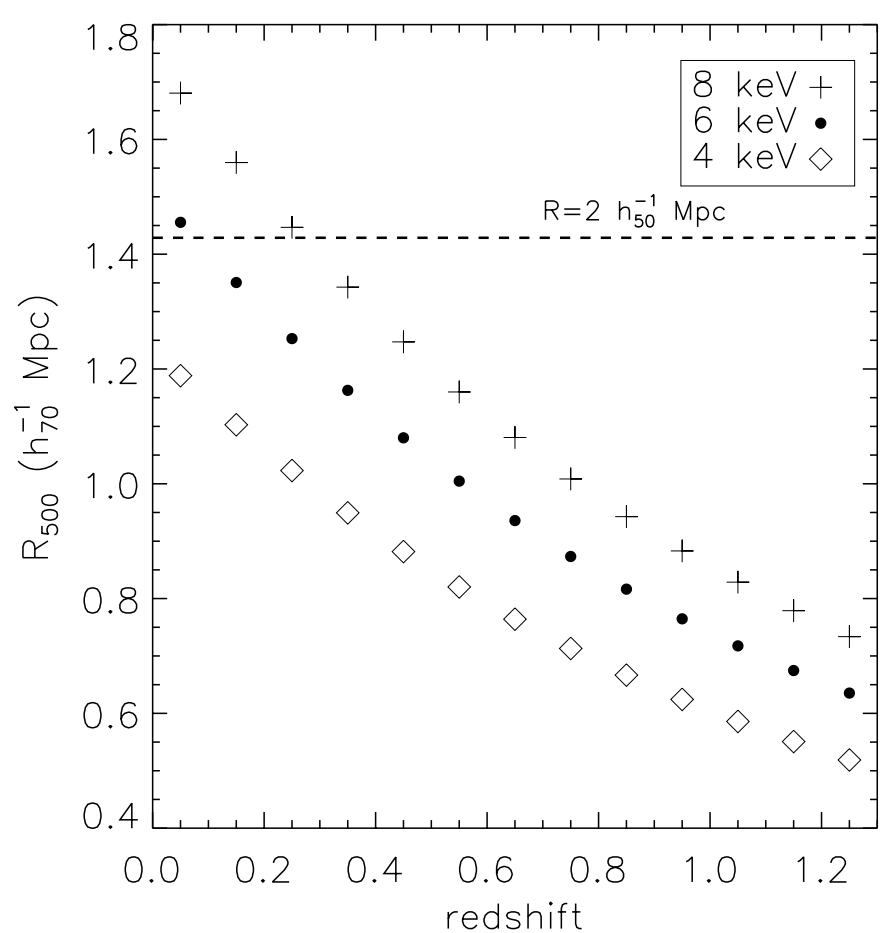

Fig. 2. Values of $R_{500}$ as function of redshift for the cosmological model assumed. The conversion from temperature (mass) to $R_{\Delta}$ is obtained by using the best-fit results of the $M-T$ relation (see Sect. 3) and Eq. (3). As comparison of what is adopted in other similar work (e.g. Vikhlinin et al. 2002), we draw the value corresponding to a fixed radius of $2 h_{50}^{-1} \mathrm{Mpc}$ (dashed line).

quantities considered in this work, i.e. total mass, gas mass and luminosity to $R_{\Delta}=R_{500}$. Specifically, an overdensity of 500 is assumed for an Einstein-de Sitter universe, whereas $\Delta_{z}=500 \times\left[1+82\left(\Omega_{z}-1\right) /\left(18 \pi^{2}\right)-39\left(\Omega_{z}-1\right)^{2} /\left(18 \pi^{2}\right)\right]$, with $\Omega_{z}=\Omega_{\mathrm{m}}(1+z)^{3} / E_{z}^{2}$, is assumed accordingly to the approximation of the solution for the collapse of a spherical tophat perturbation that just virialized at the observed redshift $z$ (e.g. Bryan \& Norman 1998). Figure 2 shows the dependence of $R_{500}$ upon the typical mass (temperature) and redshift values spanned by the clusters in our sample. Changes of about 80 per cent are expected for $R_{500}$ in the redshift range 0.4 and 1.3 , whereas a fixed metric radius remains constant by definition.

In this work, the luminosity within $R_{\Delta}$ is then estimated by correcting the value measured directly from the spectrum within $R_{\text {spec }}$ as follows

$L\left(<R_{\Delta}\right)=L\left(<R_{\mathrm{spec}}\right) \frac{\int_{0}^{R_{\Delta}}\left(1+x^{2}\right)^{-3 \beta} x^{2} \mathrm{~d} x}{E I}$,

where $E I$ is given in Eq. (2). No further corrections on the measurements of the luminosity are applied, i.e. no excess from any centrally peaked surface brightness due to the presence of "cooling flows" is excised. On the other hand, the lack of any evidence for a significant excess of the brightness in the core regions, as discussed above, indicates that no relevant differences in the total luminosity are expected when excision is applied (e.g. Vikhlinin et al. 2002) as we also show at the end of this section. 
Table 1. Best-fit results of the spectral and spatial analysis of the sample of galaxy clusters at redshift $>0.4$ and derived quantities at an overdensity corresponding to $\Delta_{z}=500$ in a Einstein-de Sitter universe. A Hubble constant of $70 \mathrm{~km} \mathrm{~s}^{-1} \mathrm{Mpc}^{-1}$ in a flat universe with $\Omega_{\mathrm{m}}$ equals to 0.3 is assumed. Note: (i) RX J1347-1145 has a clear hot enhancement to the South-East of the main X-ray emission. A sector with position angle $90^{\circ}-180^{\circ}$ and centered on the X-ray peak has been masked in accordance with the analysis presented in Allen et al. (2002). (ii) In 3C 295, the central active galactic nucleus and regions of enhanced emission associated to the radio lobes have been masked (see Allen et al. 2001a). (iii) MS1054-0321 presents significant substructure (e.g. Jeltema et al. 2001). The temperature and the best-fit of the surface brightness profile are estimated from the main body of the cluster once a circular region centered at (RA, Dec; 2000$)=\left(10^{\mathrm{h}} 56^{\mathrm{m}} 55^{\prime \prime} 7,-3^{\circ} 37^{\prime} 37^{\prime \prime}\right)$ and with radius of 36 arcsec is masked.

\begin{tabular}{|c|c|c|c|c|c|c|c|c|c|c|c|}
\hline uster & $z$ & $\begin{array}{c}1^{\prime} \\
\mathrm{kpc}\end{array}$ & $\begin{array}{r}R_{\text {spec }} \\
\mathrm{kpc}\end{array}$ & $\begin{array}{l}R_{2 \sigma} \\
\mathrm{kpc}\end{array}$ & $\begin{array}{l}T_{\text {gas }} \\
\mathrm{keV}\end{array}$ & $\begin{array}{c}r_{\mathrm{c}} \\
\mathrm{kpc}\end{array}$ & $\beta$ & $\begin{array}{l}R_{500} \\
\mathrm{kpc}\end{array}$ & $\begin{array}{c}L_{\mathrm{bol}} \\
10^{44} \mathrm{erg} \mathrm{s}^{-1}\end{array}$ & $\begin{array}{c}M_{\text {gas }} \\
10^{13} M_{\odot}\end{array}$ & $\begin{array}{c}M_{\mathrm{tot}} \\
10^{14} M_{\odot}\end{array}$ \\
\hline RX J1416+4446 & 0.400 & 322 & 397 & 1088 & $3.7_{-0.3}^{+0.2}$ & $26( \pm 4)$ & $0.438( \pm 0.012)$ & $749( \pm 26)$ & $5.43( \pm 0.19)$ & $2.84( \pm 0.47)$ & $1.35( \pm 0.14)$ \\
\hline MS0302+1658 & 0.424 & 334 & 274 & 552 & $3.8_{-0.2}^{+0.9}$ & $64( \pm 10)$ & $0.630( \pm 0.036)$ & $899( \pm 65)$ & $6.41( \pm 0.19)$ & $3.26( \pm 0.48)$ & $2.43( \pm 0.55)$ \\
\hline MS1621+2640 & 0.426 & 335 & 659 & 1276 & $6.8_{-0.5}^{+0.9}$ & $185( \pm 19)$ & $0.563( \pm 0.040)$ & $1119( \pm 74)$ & $10.92( \pm 0.30)$ & $7.18( \pm 0.76)$ & $4.70( \pm 0.94)$ \\
\hline RX J1347-1145 & 0.451 & 346 & 533 & 1430 & $10.3_{-0.5}^{+0.6}$ & $38( \pm 1)$ & $0.571( \pm 0.004)$ & $1368( \pm 40)$ & $116.75( \pm 0.58)$ & $18.06( \pm 0.80)$ & $8.94( \pm 0.80)$ \\
\hline RX J1701+6421 & 0.453 & 347 & 370 & 889 & $4.5_{-0.2}^{+0.4}$ & $13( \pm 2)$ & $0.396( \pm 0.007)$ & $750( \pm 24)$ & $6.42( \pm 0.58)$ & $3.36( \pm 0.45)$ & $1.48( \pm 0.15)$ \\
\hline 3C 295 & 0.460 & 350 & 172 & 895 & $4.3_{-0.2}^{+0.3}$ & $31( \pm 2)$ & $0.553( \pm 0.007)$ & $870( \pm 26)$ & $14.07( \pm 0.19)$ & $4.35( \pm 0.29)$ & $2.33( \pm 0.21)$ \\
\hline RX J1525+0957 & 0.516 & 372 & 372 & 973 & $5.1_{-0.5}^{+0.5}$ & $229( \pm 30)$ & $0.644( \pm 0.054)$ & $941( \pm 60)$ & $6.92( \pm 0.19)$ & $4.74( \pm 0.62)$ & $3.23( \pm 0.62)$ \\
\hline MS0451-0305 & 0.539 & 381 & 625 & 1391 & $8.0_{-0.3}^{+0.3}$ & $201( \pm 6)$ & $0.734( \pm 0.015)$ & $1263( \pm 30)$ & $50.94( \pm 8.05)$ & $14.15( \pm 0.47)$ & $8.09( \pm 0.59)$ \\
\hline MS0016+1609 & 0.541 & 382 & 626 & 1770 & $10.0_{-0.5}^{+0.5}$ & $237( \pm 8)$ & $0.685( \pm 0.013)$ & $1355( \pm 35)$ & $53.27( \pm 7.33)$ & $17.13( \pm 0.66)$ & $10.02( \pm 0.78)$ \\
\hline RX J1121+2326 & 0.562 & 389 & 446 & 702 & $4.6_{-0.3}^{+0.5}$ & $427( \pm 85)$ & $1.180( \pm 0.276)$ & $1132( \pm 128)$ & $5.45( \pm 0.22)$ & $4.60( \pm 0.39)$ & $6.04( \pm 2.12)$ \\
\hline $8+4456$ & 0.570 & 391 & 196 & 401 & $3.2_{-0.3}^{+0.3}$ & $97( \pm 14)$ & $0.620( \pm 0.050)$ & $723( \pm 45)$ & $1.21( \pm 0.37)$ & $1.23( \pm 0.14)$ & $1.59( \pm 0.30)$ \\
\hline MS20 & 0.583 & 396 & 357 & 730 & $5.5_{-0.5}^{+0.5}$ & $99( \pm 11)$ & $0.610( \pm 0.033)$ & $931( \pm 47)$ & $5.40( \pm 1.04)$ & $3.41( \pm 0$ & $3.47( \pm 0$ \\
\hline RX J0 & 0.634 & 411 & 540 & 1198 & $7.9_{-0.8}^{+1.0}$ & $132( \pm 17)$ & $0.514( \pm 0.031)$ & $982( \pm 63)$ & $12.15( \pm 1.36)$ & $6.27( \pm 0.82)$ & $4.41( \pm$ \\
\hline $\mathrm{RX} \mathrm{J12}$ & 0.700 & 429 & 563 & 972 & $7.5_{-0.6}^{+0.7}$ & $263( \pm 22)$ & $0.734( \pm 0.047)$ & $1063( \pm 57)$ & $12.95( \pm 0.39)$ & $6.80( \pm 0.59)$ & $6.16( \pm 1.00)$ \\
\hline RX J11 & 0.730 & 436 & 286 & 371 & $5.6_{-0.6}^{+0.0}$ & $89( \pm 13)$ & $0.639( \pm 0.049)$ & $866( \pm 62)$ & $4.43( \pm 0.77)$ & $2.49( \pm 0$ & $3.47( \pm 0.75)$ \\
\hline RX J2302+0844 & 0.734 & 437 & 358 & 635 & $6.6_{-0.6}^{+1.5}$ & $96( \pm 12)$ & $0.546( \pm 0.033)$ & $865( \pm 65)$ & $5.45( \pm 0.17)$ & $3.38( \pm 0.48)$ & $3.48( \pm 0.82)$ \\
\hline MS1137+6625 & 0.782 & 447 & 367 & 635 & $6.9_{-0.5}^{+0.0}$ & $111( \pm 6)$ & $0.705( \pm 0.022)$ & $964( \pm 39)$ & $15.30( \pm 0.44)$ & $4.90( \pm 0.29)$ & $5.18( \pm 0.63)$ \\
\hline $\mathrm{RX} \mathrm{J} 1$ & 0.805 & 451 & 185 & 238 & $4.1_{-0.8}^{+1.2}$ & $50( \pm 12)$ & $0.518( \pm 0.048)$ & $634( \pm 83)$ & $1.12( \pm 0.08)$ & $0.98( \pm 0.29)$ & $1.52( \pm 0.61)$ \\
\hline RX J1350+6007 & 0.810 & 452 & 519 & 801 & $4.6_{-0.9}^{+0.7}$ & $106( \pm 37)$ & $0.479( \pm 0.056)$ & $627( \pm 65)$ & $4.41( \pm 0.39)$ & $2.24( \pm 0.79)$ & $1.48( \pm 0.46)$ \\
\hline RX J1716+6708 & 0.813 & 453 & 371 & 640 & $6.8_{-0.6}^{+1.0}$ & $121( \pm 16)$ & $0.635( \pm 0.038)$ & $896( \pm 56)$ & $13.86( \pm 1.04)$ & $4.87( \pm 0.61)$ & $4.35( \pm 0.83)$ \\
\hline MS1054-0321 & 0.830 & 456 & 628 & 900 & $10.2_{-0.8}^{+1.0}$ & $511( \pm 36)$ & $1.375( \pm 0.124)$ & $1509( \pm 93)$ & $28.48( \pm 2.96)$ & $10.61( \pm 0.44)$ & $21.27( \pm 3.96)$ \\
\hline $1357 \mathrm{~S}$ & 0.830 & 456 & 400 & 607 & & $96( \pm 19)$ & $0.532( \pm 0.046)$ & $824( \pm 109)$ & $7.73( \pm 0.40)$ & $3.91( \pm 0.94)$ & $3.47( \pm 1.49)$ \\
\hline RX J0152-1357N & 0.835 & 457 & 427 & 919 & $6.0_{-0.7}^{+1.1}$ & $157( \pm 36)$ & $0.542( \pm 0.067)$ & $753( \pm 72)$ & $10.67( \pm 0.67)$ & $4.49( \pm 1.01)$ & $2.66( \pm 0.77)$ \\
\hline WGA1226+3333 & 0.890 & 466 & 459 & 711 & $11.2_{-1.5}^{+2.2}$ & $123( \pm 13)$ & $0.692( \pm 0.037)$ & $1134( \pm 95)$ & $54.63( \pm 0.83)$ & $10.54( \pm 1.33)$ & $9.80( \pm 2.55)$ \\
\hline RX J0910+5422 & 1.106 & 491 & 201 & 273 & $6.6_{-1.3}^{+1.7}$ & $147( \pm 53)$ & $0.843( \pm 0.268)$ & $818( \pm 150)$ & $2.83( \pm 0.35)$ & $1.56( \pm 0.30)$ & $4.91( \pm 2.93)$ \\
\hline RX J1252-2927 & 1.235 & 500 & 492 & 440 & $5.2_{-0.7}^{+0.7}$ & $77( \pm 13)$ & $0.525( \pm 0.034)$ & $532( \pm 40)$ & $5.99( \pm 1.10)$ & $1.81( \pm 0.34)$ & $1.59( \pm 0.35)$ \\
\hline RX J0849+4452 & 1.260 & 501 & 197 & 275 & $5.2_{-1.1}^{+1.6}$ & $128( \pm 40)$ & $0.773( \pm 0.189)$ & $640( \pm 106)$ & $2.83( \pm 0.17)$ & $1.38( \pm 0.30)$ & $2.85( \pm 1.48)$ \\
\hline RX J0848+4453 & 1.270 & 501 & 164 & 173 & $2.9_{-0.8}^{+0.8}$ & $163( \pm 70)$ & $0.972( \pm 0.431)$ & $499( \pm 115)$ & $1.04( \pm 0.73)$ & $0.56( \pm 0.21)$ & $1.37( \pm 0.98)$ \\
\hline
\end{tabular}

The quoted errors on luminosity are obtained through the propagation of the errors obtained in XSPEC from the distribution around the best-fit values in the spectral analysis (which are the dominant contribution), and the uncertainties related to the $\beta$-model in moving from $R_{\text {spec }}$ to $R_{\Delta}$.

In the Appendix, we show the plots of the surface brightness profiles of our clusters, along with the $\beta$-model obtained through a least-squares fitting process and the relative positions of $R_{\Delta}, R_{\text {spec }}$ and $R_{2 \sigma}$. The latter radius indicates up to where the signal is above $2 \sigma$ with respect to the best-fit value of the background and gives an indication of the region of the cluster where a diffuse emission is detectable.

Out of 28 clusters, 17 have $R_{2 \sigma}$ that is smaller than $R_{500}$ by a factor between 1.1 and 3.0 (median of 1.4) and need extrapolation to compute their quantities. The spectral aperture, $R_{\mathrm{spec}}$, corresponds roughly to an overdensity of $\sim 2000$ and is about $0.6 R_{2 \sigma}$ and 3.6 times larger than the cluster core radius (median values). It is worth noticing that more than 75 per cent of the cluster luminosity, on average, is however sampled within $R_{\text {spec }}$ because of the $n_{\text {gas }}^{2}$ dependence of the X-ray thermal emission.

Finally, we compare our estimates of $T_{\text {gas }}$ and $L_{\text {bol }}$ with the values quoted by Vikhlinin et al. (2002). These authors have 21 clusters in common with us (including also CL0024 that is not listed here because at redshift 0.395 and, therefore, below our cut at $z=0.4$ ). The agreement is remarkably good, with a median ratio between our and Vikhlinin et al. measurements of temperature of 1.04 (mean: 1.08; see Fig. 3) and estimates of luminosity (at $2 h_{50}^{-1} \mathrm{Mpc}$ ) of 1.00 (1.08 in average). Larger $(>|2 \sigma|$ when comparing our values vs. Vikhlinin et al. ones) deviations are observed in the temperature estimates of RX J1347 $(-4.7 \sigma)$, RX J1701 $(-3.0 \sigma)$, CL 0024 $(-2.3 \sigma), 3 \mathrm{C} 295(-2.3 \sigma)$ and MS1054 $(+2.5 \sigma)$, and in the luminosity measurements of 3C $295(+8.2 \sigma)$, RX J1416 $(+6.2 \sigma)$, RX J1317 $(+3.1 \sigma)$ and MS0302 $(+2.3 \sigma)$. We notice that all 

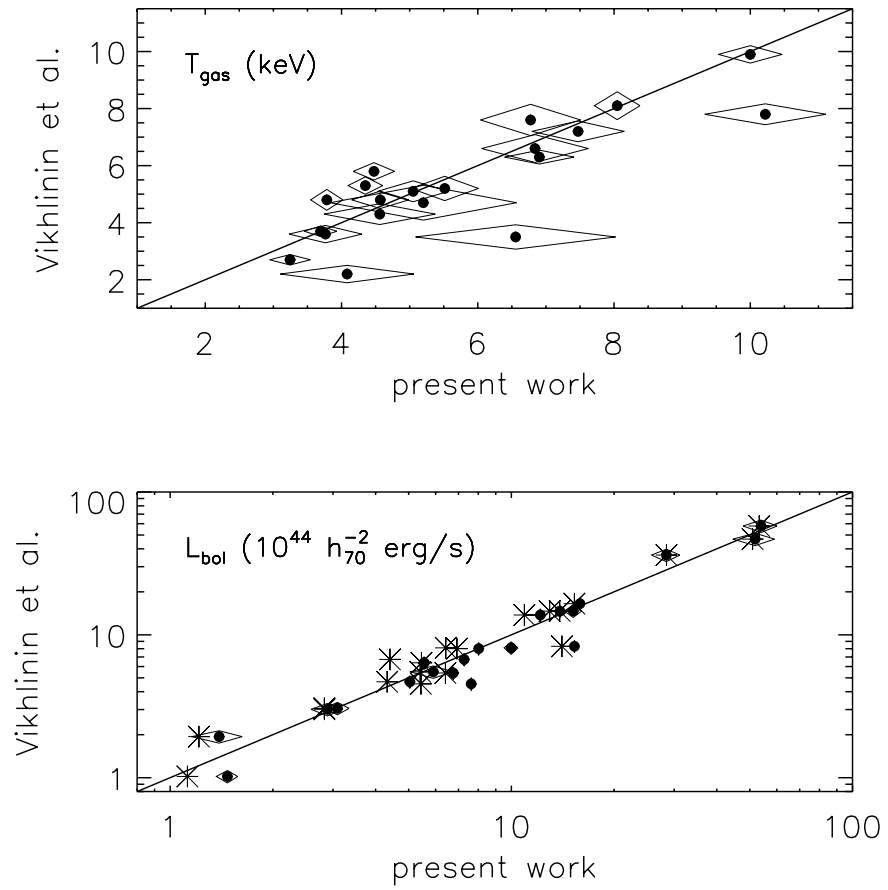

Fig. 3. Comparison between the temperatures and luminosities estimated in the present work and by Vikhlinin et al. (2002) for the 21 clusters that we have in common. (Upper panel) A median (mean) difference of 4 (8) per cent is present between our measurements of gas temperature and the values quoted in Vikhlinin et al. (Lower panel) The dots indicate the estimates within $2 h_{50}^{-1} \mathrm{Mpc}$, whereas the asterisks show our measurements of $L_{\mathrm{bol}}\left(<R_{500}\right)$. A median (mean) difference of 0 (8) per cent is observed between our values and the ones in Vikhlinin et al.

these objects (apart from MS1054 that presents a significant substructure that we mask out in our analysis as described in the caption of Table 1) are classified by Vikhlinin and collaborators as systems with a "possible cooling core" (even though they do not describe how they quantify the sharpener of the central brightness). The core emission is then excised by Vikhlinin et al. during the process of the determination of the gas temperature and luminosity, whereas we maintain it in our procedure.

\section{The scaling relations at $0.4<z<1.3$}

A sensible way to test the hierarchical formation of galaxy clusters and the influence of non-gravitational processes on their self-similarity at different epochs is to study how the scaling relations behave and evolve at high redshifts. In practice, by using the observed $(L, T)$ and derived $\left(M_{\mathrm{tot}}, M_{\mathrm{gas}}\right)$ physical properties measured within a given overdensity $\Delta_{z}$ with respect to the critical overdensity $\rho_{\mathrm{c}, \mathrm{z}}$, one wants to investigate whether the quantities $E_{z} M_{\text {tot }} / T_{\text {gas }}^{3 / 2}$ and $E_{z}^{-1} L_{\text {bol }} / T_{\text {gas }}^{2}$ (and the complementary ones, $E_{z} M_{\text {gas }} / T_{\text {gas }}^{3 / 2}$ and $\left.E_{z}^{-1} L_{\text {bol }} /\left(E_{z} M_{\text {tot }}\right)^{4 / 3}\right)$ show, or not, any evolution with redshift and/or dependence upon one of the physical properties, typically the temperature.

The behavior of these scaling laws are examined first in their normalization and slope by fitting the logarithmic relation

$\log Y=\alpha+A \log X$
Table 2. Mean, median and dispersion values for the best-fit parameters $r_{\mathrm{c}}$ and $\beta$ estimated for objects in the chosen redshift bins.

\begin{tabular}{lcc}
\hline \hline range $z$ & $\begin{array}{c}r_{\mathrm{c}} \\
h_{70}^{-1} \mathrm{kpc}\end{array}$ & $\beta$ \\
\hline $0.4-0.6$ & $98,137(162)$ & $0.615,0.635(0.217)$ \\
$0.6-0.8$ & $111,138(76)$ & $0.639,0.627(0.096)$ \\
$0.8-1.0$ & $121,166(166)$ & $0.542,0.682(0.324)$ \\
$>1.0$ & $137,128(61)$ & $0.808,0.778(0.333)$ \\
\hline
\end{tabular}

between two sets of measured quantities $\left\{X_{j}\right\}$ and $\left\{Y_{j}\right\}$. We use the bisector modification (i.e. the best-fit results bisect those obtained from minimization in vertical and horizontal directions) of the linear regression algorithm in Akritas \& Bershady (1996 and references therein, hereafter BCES) that takes into account both any intrinsic scatter and errors on the two variables considered as symmetric. The uncertainties on the bestfit results are obtained from 10000 bootstrap resampling. The results on the best-fit normalization and slope for the scaling laws here investigated are quoted in Table 3 and shown as dotted (when the slope is fixed to the predicted value) and dashed (when the slope is a free parameter) lines in Figs. 4-7.

In general, we observe a steeper slope than expected from self-similar model in all the scaling laws under exam, with $L-T$ (Fig. 4) and $M_{\text {gas }}-T$ (Fig. 6) relations being the ones with deviations larger than $3 \sigma$ (best-fit of $A=3.72 \pm 0.47$ and $2.37 \pm 0.24$, respectively), whereas $M_{\text {tot }}-T$ (Fig. 5) and $L-M_{\text {tot }}$ (Fig. 7) deviates by about $1.5 \sigma$ from the expected values of $A=1.5$ and 1.33 . This is a trend usually well-observed at lower redshift and that affects all the quantities depending directly on the measurements of the amount of gas, like luminosity and gas mass (e.g. Edge \& Stewart 1991; Fabian et al. 1994; Mushotzky \& Scharf 1997; Allen \& Fabian 1998; Mohr et al. 1999; Novicki et al. 2001; McCarthy et al. 2002; Ettori et al. 2002). This confirms that simple gravitational collapse is not the only process that governs the accretion of baryons in the potential well, but some combination of properly efficient cooling and extra heating is required to take place before or during the hierarchical formation of clusters (Kaiser 1991; Evrard \& Henry 1991; Cavaliere et al. 1999; Tozzi \& Norman 2001; Balogh et al. 2002; Voit et al. 2002; Tornatore et al. 2003). Scaling the X-ray luminosity as $L \propto n_{\text {gas }}^{2} T^{2} R^{3} \sim f_{\text {gas }}^{2} T^{2}$, David et al. (1993) suggested that if the gas mass fraction, $f_{\mathrm{gas}}=M_{\mathrm{gas}} / M_{\mathrm{tot}}$, is proportional to $T^{0.5}$, the steepening of the $L-T$ relation can be explained. However, we rule out any statistically significant dependence of $f_{\text {gas }}$ on the gas temperature in two ways. First, we measure a probability of 66 per cent that no-correlation is present in the distribution of the values in the plane $f_{\text {gas }}-T$ once the Spearman's rank coefficient is estimated (by fitting a power-law to the data, we measure a slope of $0.5 \pm 1.0$ ). Then, we try to constrain the best-fit parameters of the model $L \sim T^{A 1}\left(M_{\mathrm{gas}} / M_{\mathrm{tot}}\right)^{A 2}$ given the estimated gas luminosities, gas masses and total gravitating masses. As shown in Fig. 8, $A 1$ is consistent with a value of 3 and different from 2 by $\sim 3 \sigma$, whereas $A 2$ is in agreement with the predicted value of 2 within $1 \sigma\left(A 2=2.48_{-0.70}^{+0.42}\right)$. 

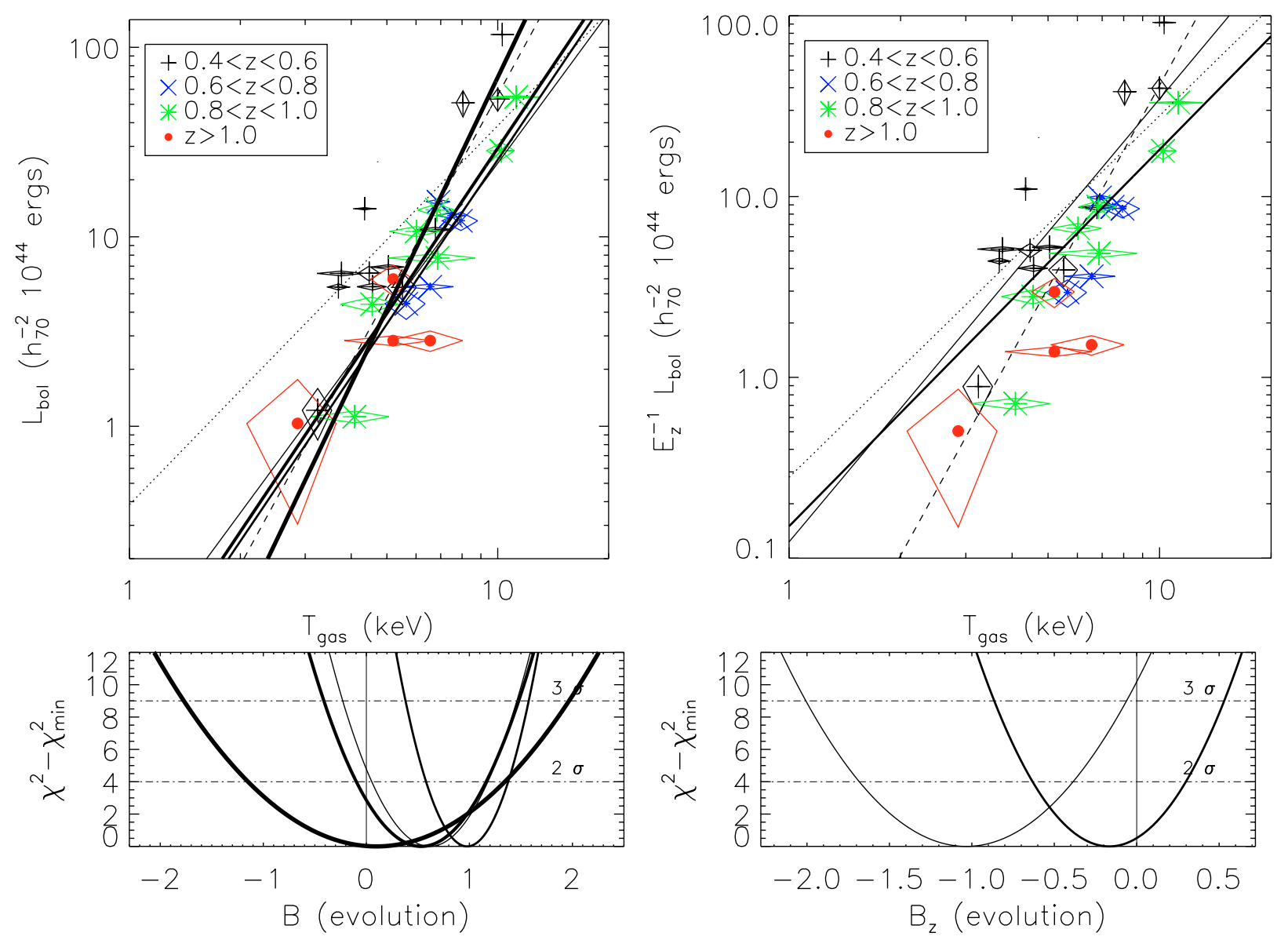

Fig. 4. $L-T$ relation without (left) and with (right) correction by $E_{z}$. (Upper panels) Dotted line: slope fixed to the predicted value of 2 . Dashed line: slope free. The solid lines represent the local best-fit results (from thinnest to thickest line): Markevitch (1998; with core excised in cooling flows clusters), Arnaud \& Evrard (1999), Novicki et al. (2002; for objects at $z<0.3$ ) and Novicki et al. (2002; for objects at $0.6>z>0.3$ ). Solid line: Ettori et al. (2002; thinest line), Allen et al. (2001c). (Bottom panels) Plot of the $\Delta \chi^{2}$ distribution for the one interesting parameter $B\left(B_{z}\right)$ given in Eq. (7). Each solid line corresponds to a local scaling relation plotted with the same thickness in the upper panel and compared to all the 28 objects with $z \geq 0.4$ in our sample. The 2 and $3 \sigma$ limits are shown as dot-dashed lines.

Table 3. Best-fit results on the scaling relations corrected by the cosmological factor $E_{z}$ (dashed and dotted lines in the plots; see Eq. (5)). The temperature, $T_{\mathrm{gas}, 6}$, is in unit of $6 \mathrm{keV}$; the luminosity, $L_{\mathrm{bol}, 44}$, in $10^{44} h_{70}^{-2} \mathrm{erg} \mathrm{s}^{-1}$; the total mass, $M_{\mathrm{tot}, 14}$, in $10^{14} h_{70}^{-1} M_{\odot}$; the gas mass, $M_{\text {gas, } 13}$, in $10^{13} h_{70}^{-5 / 2} M_{\odot}$. When the slope $A$ is fixed, we estimate the error-weighted mean of $(\log Y-A \log X)$ and evaluate the error after resampling $Y$ and $X$ by 1000 times according to their uncertainties. The scatter on $Y$ is measured as $\left[\sum_{j=1, N}\left(\log Y_{j}-\alpha-A \log X_{j}\right)^{2} / N\right]^{1 / 2}$. Note that the scatter along the $X$-axis can be estimated as $\sigma_{\log X}=$ $\sigma_{\log Y} / A$.

\begin{tabular}{lccc}
\hline \hline Relation $(Y-X)$ & $\alpha$ & $A$ & $\sigma_{Y}$ \\
\hline$E_{z}^{-1} L_{\mathrm{bol}, 44}-T_{\text {gas }, 6}$ & $0.79( \pm 0.07)$ & $3.72( \pm 0.47)$ & 0.35 \\
& $1.00( \pm 0.02)$ & 2.00 (fixed) & 0.41 \\
$E_{z} M_{\mathrm{tot}, 14}-T_{\text {gas }, 6}$ & $0.75( \pm 0.03)$ & $1.98( \pm 0.30)$ & 0.15 \\
& $0.74( \pm 0.02)$ & $1.50($ fixed $)$ & 0.15 \\
$E_{z} M_{\text {gas }, 13}-T_{\text {gas }, 6}$ & $0.79( \pm 0.03)$ & $2.37( \pm 0.24)$ & 0.17 \\
& $0.92( \pm 0.01)$ & 1.50 (fixed) & 0.22 \\
$E_{z}^{-1} L_{\text {bol }, 44}-E_{z} M_{\text {tot }, 14}$ & $-0.63( \pm 0.32)$ & $1.88( \pm 0.42)$ & 0.47 \\
& $0.09( \pm 0.03)$ & 1.33 (fixed) & 0.52 \\
\hline
\end{tabular}

As originally pointed out by Horner et al. (1999) and Nevalainen et al. (2000), samples of nearby clusters indicate that systems at given temperature are systematically less massive than the counterparts measured in numerical simulations (e.g. Evrard et al. 1996; Mathiesen \& Evrard 2001; Borgani et al. 2002), where normalizations of the $M_{\mathrm{tot}}-T$ relation are larger by about 30 per cent or more than observed, once the slope is fixed to the expected value of $3 / 2$. We confirm this result at $z>0.4$, where we measure a normalization of $(0.37 \pm 0.02) \times 10^{14} h_{70}^{-1} M_{\odot} \mathrm{keV}^{-3 / 2}$, that is indeed $\sim 30$ per cent lower than what obtained in the hydrodynamical simulations of Mathiesen \& Evrard (2001; see Fig. 5), but well in agreement with previous observational results obtained for cluster samples at lower redshifts (e.g. Nevalainen et al. 2000; Allen et al. 2001c).

We note that the observed scatter in these relations indicates a correlation between total/gas mass and gas temperature, which is as tight at high redshift as measured locally. This suggests that a marginal role is played by evolutionary effects, such as merging history and non-gravitational heating, during the assembly of baryonic and dark matter into the clusters. A larger scatter is present in the $L-T$ and $L-M_{\text {tot }}$ relations. This is just 

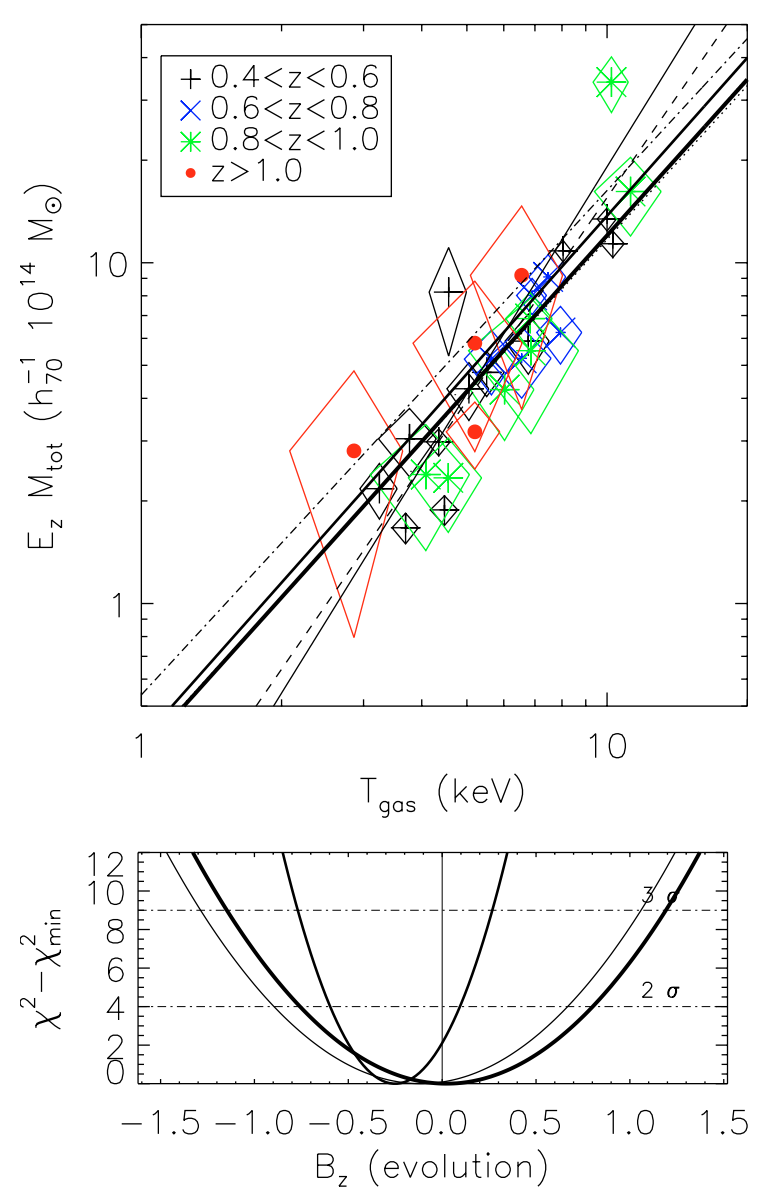

Fig. 5. $M_{\mathrm{tot}}-T$ relation. (Upper panels) Dotted line: slope fixed to the predicted value of $3 / 2$. Dashed line: slope free. The solid lines represent the local best-fit results (from thinnest to thickest line): Ettori et al. (2002), Finoguenov et al. (2001), Allen et al. (2001c). The dashdot line indicates the best-fit results from hydrodynamical simulations in Mathiesen \& Evrard (2001, Table 1, with temperatures from simulated spectral analysis in the band $0.5-9.5 \mathrm{keV}$ ). (Bottom panels) Plot of the $\Delta \chi^{2}$ distribution for the one interesting parameter $B_{z}$ given in Eq. (7). Each solid line corresponds to a local scaling relation plotted with the same thickness in the upper panel and compared to all the 28 objects with $z \geq 0.4$ in our sample. The 2 and $3 \sigma$ limits are shown as dot-dashed lines.

the consequence of the higher sensitivity of X-ray luminosity to the details of the gas distribution and how it is affected by both gravitational dynamics and non-gravitational processes.

\section{On the evolution of the scaling laws}

To constrain the evolution in the considered scaling laws, we fix $(\alpha, A)$ to the best-fit results obtained from a sample of objects observed at lower redshift, $(\bar{\alpha}, \bar{A})$, and evaluate the confidence interval through a least-square minimization on the parameter $B$ in the relation

$\log Y=\bar{\alpha}+\bar{A} \log X+B \log (1+z)$
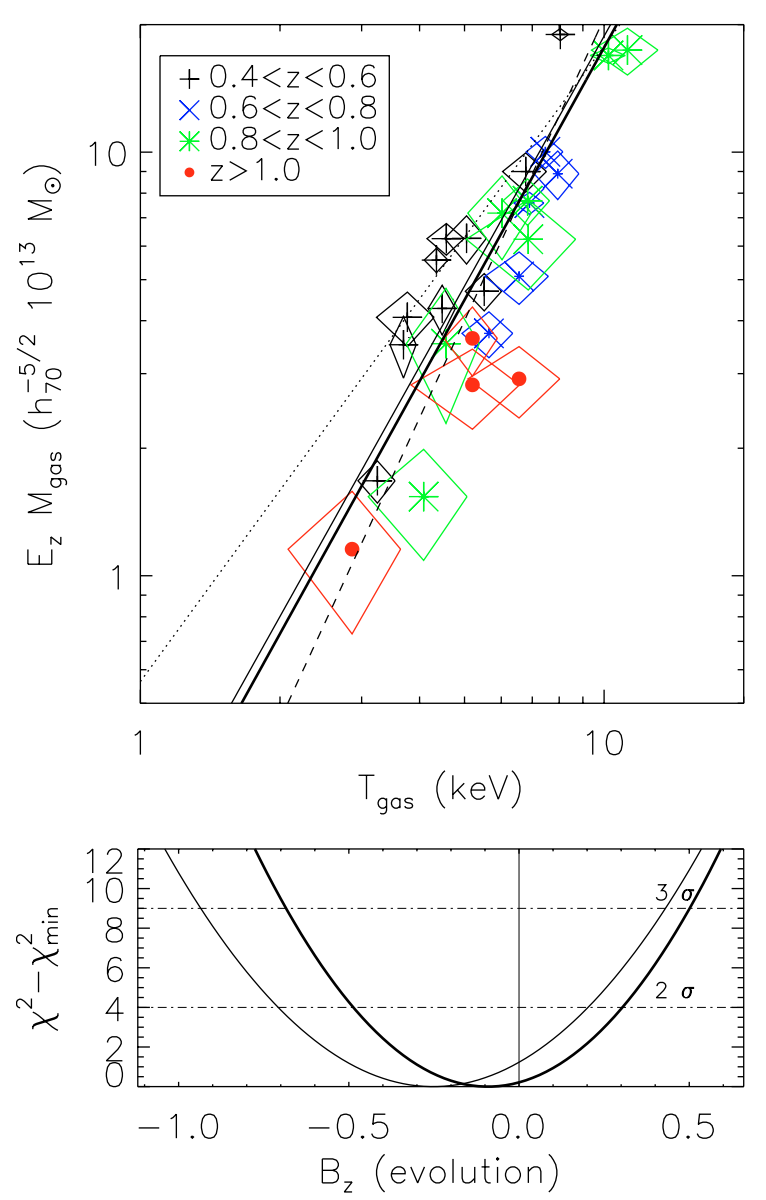

Fig. 6. $M_{\text {gas }}-T$ relation. (Upper panels) Dotted line: slope fixed to the predicted value of $3 / 2$. Dashed line: slope free. The solid lines represent the local best-fit results (from thinnest to thickest line): Ettori et al. (2002), Mohr et al. (1999). (Bottom panels) Plot of the $\Delta \chi^{2}$ distribution for the one interesting parameter $B_{z}$ given in Eq. (7). Each solid line corresponds to a local scaling relation plotted with the same thickness in the upper panel and compared to all the 28 objects with $z \geq 0.4$ in our sample. The 2 and $3 \sigma$ limits are shown as dot-dashed lines.

In particular, for a given grid of values of $\left\{B_{i}\right\}$, we search for the minimum of the merit function

$\chi_{i}^{2}=\sum_{j} \frac{\left[\log Y_{j}-\bar{\alpha}-\bar{A} \log X_{j}-B_{i} \log \left(1+z_{j}\right)\right]^{2}}{\epsilon_{\log Y_{j}}^{2}+\epsilon_{\alpha}^{2}+\bar{A}^{2} \epsilon_{\log X_{j}}^{2}+\epsilon_{\bar{A}}^{2} \log ^{2} X_{j}}$,

where the errors on the best-fit local values, $\epsilon_{\bar{\alpha}}$ and $\epsilon_{\bar{A}}$, are considered and propagated with the uncertainties, $\epsilon_{\log X}=\epsilon_{X} /(X \ln 10)$ and $\epsilon_{\log Y}=\epsilon_{Y} /(Y \ln 10)$, on the measured quantities.

The local and intermediate-redshift relations considered in this work have been rescaled to the assumed cosmology and overdensity as described in the Introduction. On the other hand, many results on the clusters scaling laws, and particularly on the $L-T$ relation, are presented in literature with the omission of the cosmological factor $E_{z}$ (that should be properly considered only when the quantities are measured within a fixed overdensity with respect to the critical density as done in the present work). Therefore, we have considered in the following analysis both the inclusion and the omission of the factor $E_{z}$ to correct 

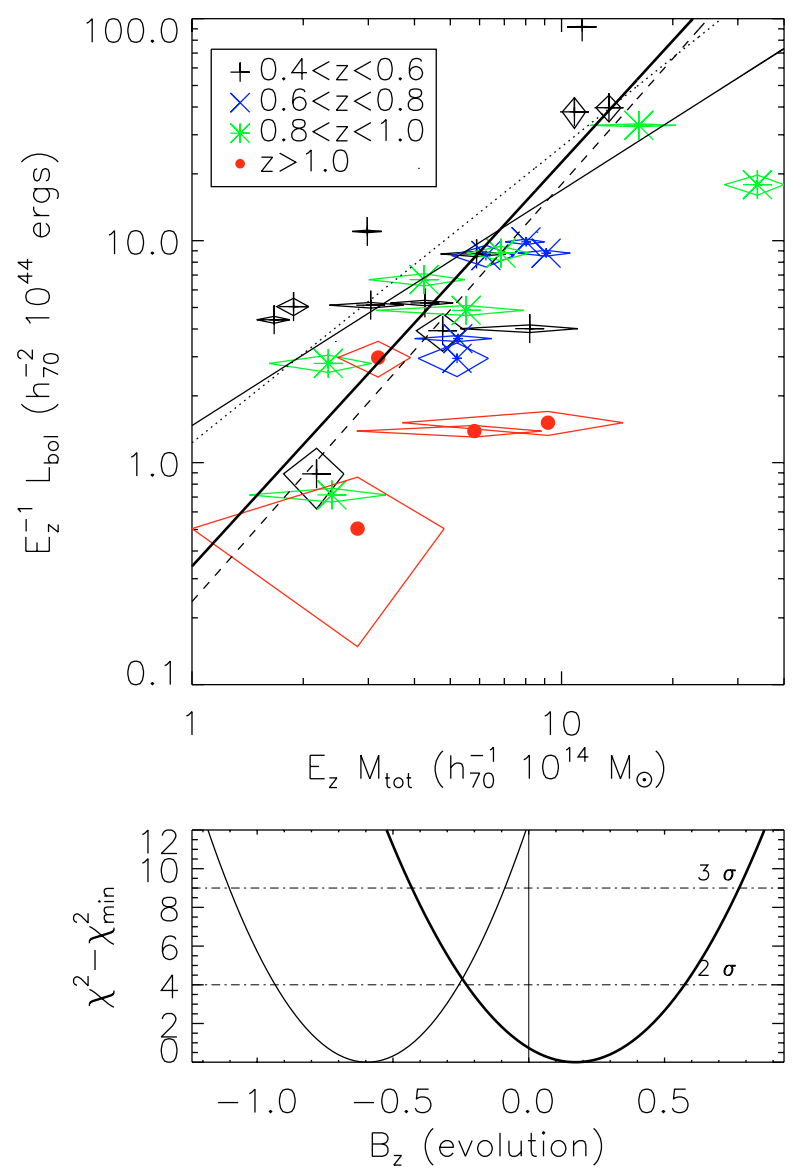

Fig. 7. $L-M_{\mathrm{tot}}$ relation. (Upper panels) Dotted line: slope fixed to the predicted value of $4 / 3$. Dashed line: slope free. The solid lines represent the local best-fit results (from thinnest to thickest line): Ettori et al. (2002), Reiprich \& Böhringer (2002). (Bottom panels) Plot of the $\Delta \chi^{2}$ distribution for the one interesting parameter $B_{z}$ given in Eq. (7). Each solid line corresponds to a local scaling relation plotted with the same thickness in the upper panel and compared to all the 28 objects with $z \geq 0.4$ in our sample. The 2 and $3 \sigma$ limits are shown as dotdashed lines.

the quantities whose relations are investigated. In particular, we refer to the parameter $B$ for the evolution in the scaling relations not corrected by $E_{z}$ and to the parameter $B_{z}$ for the evolution in the corrected scaling laws.

It is worth noticing that the cosmological factor $E_{z}$ is defined as $H_{z} / H_{0}=\left[\Omega_{\mathrm{m}}(1+z)^{3}+1-\Omega_{\mathrm{m}}\right]^{1 / 2}$ (for a flat cosmology with matter density $\left.\Omega_{\mathrm{m}}\right)$ and is exactly equal to $(1+z)^{1.5}$ in an Einstein-de Sitter universe and proportional to $(1+z)^{0.6 / 0.9}$ in the redshift range here considered for an assumed $\Lambda \mathrm{CDM}$ model with $\Omega_{\mathrm{m}}=0.3^{1}$. Furthermore, considering its dependence upon the redshift, this factor affects significantly the quoted values of, e.g., mass and luminosity for objects at high $-z$. For example, the X-ray luminosity has to be corrected by a factor of 0.81 at $z=0.4$ and 0.51 at $z=1.2$, making

${ }^{1}$ The slope of the dependence of $E_{z}$ upon $(1+z)$ is the values of the ratio $\log E_{z} / \log (1+z)$ for an assumed cosmology and a given range in redshift.

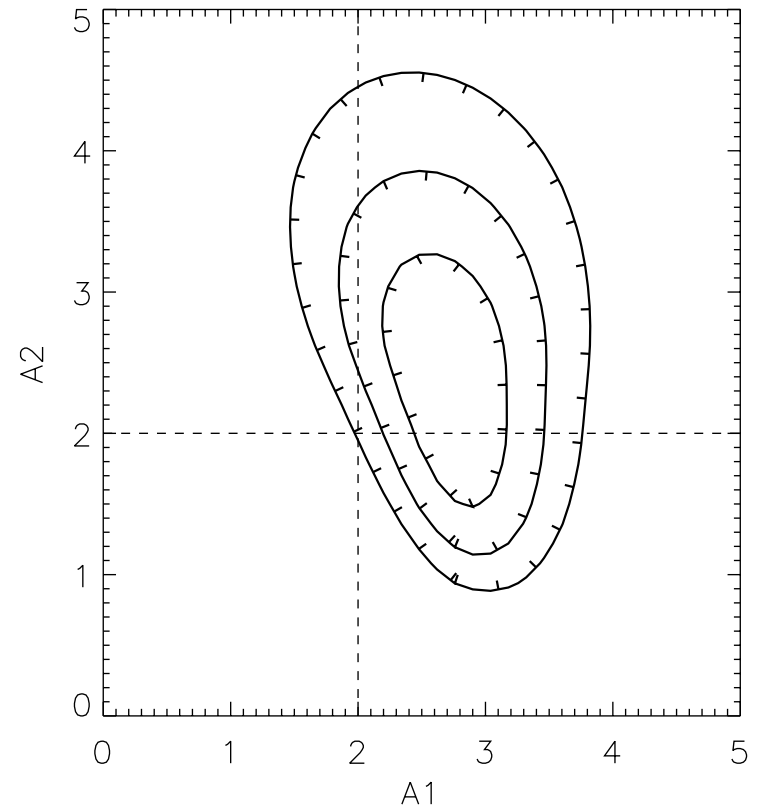

Fig. 8. Slopes in the relation $L_{\mathrm{bol}, 44}=A 0 \times T_{\mathrm{gas}, 6}^{A 1}\left(M_{\mathrm{gas}, 13} / M_{\mathrm{tot}, 14}\right)^{A 2}(1+$ $z)^{A 3}$ obtained by minimization of the $\chi^{2}$ (best-fit results: $A 0=2.0$, $A 1=2.8, A 2=1.9, A 3=0.9, \chi_{\min }^{2}=29.2$ with 24 d.o.f.) and after marginalization on the parameters $A 0$ and $A 3$. The dashed lines indicate the predicted values of 2 from the simple gravitational collapse model assuming bremsstrahlung emission.

these corrections fundamental to proper calibrate the scaling relations in a sample of clusters distributed over a wide interval in redshifts. Thus, the omission of the factor $E_{z}$, that affects each quantity in a different way at different redshift as stated above, has to be properly considered once one wants to assess any evolution of the physical quantities that is not due to the expansion of the Universe and to the rescaling of the characteristic density.

With this notation in hand, we should observe $B_{z}=0$ and $B$ equal to the expected dependence of the scaling law upon $E_{z}$ if no evolution of the physical quantities is present. In the following analysis, only the evolution of the $L-T$ relation is presented by separating $B$ and $B_{z}$. One should bear in mind that the $L-T$ relation is then proportional to $E_{z}$ implying that $B$ should be measured in the range 0.6-0.9.

We describe below the constraints that we obtain on the parameters $B$ and $B_{z}$, whose $\chi^{2}$ distribution is plotted in the bottom panel of the figure that shows the corresponding scaling relation. All the results, obtained also by selecting different redshift-range in our sample, are presented in Table 4.

\subsection{Evolution in the $L-T$ relation}

We compare the distribution of our measurements in the $L-$ $T$ plane (Fig. 4) with the best-fit results obtained for homogeneous and representative sets of nearby clusters from (i) Markevitch (1998), who analyzed a sample of 31 clusters in the redshift range 0.04-0.09 with measured ASCA temperatures and ROSAT luminosities and excises systematically their inner $100 \mathrm{kpc}$ to avoid any contamination from central cooling cores, (ii) Arnaud \& Evrard (1999) that include in their 
Table 4. The best-fit values of the evolution parameter $B, B_{z}$ (see Eqs. (6) and (7) and comments in Sect. 4) are obtained by comparing to the local best-fit results, indicated in the second column and discussed in the text, the 28, 16 and 11 clusters with redshift larger than $0.4,0.6$ and 0.8 , respectively. The case of no evolution corresponds to a value of $B_{z}$ equal to 0 . The $L-T$ relation has been studied also not including the correction by $E_{z}$. This relation is then expected to be proportional to $E_{z}$ and $B$ should be measured in the range $0.6-0.9$ in the adopted cosmology.

\begin{tabular}{lcccc}
\hline \hline Relation & Local ref. & $(z \geq 0.4)$ & $(z \geq 0.6)$ & $(z \geq 0.8)$ \\
\hline & & & $B$ & \\
(Fig. 4) & Markevitch (1998) & $0.62( \pm 0.28)$ & $0.04( \pm 0.33)$ & $0.04( \pm 0.39)$ \\
& Arnaud \& Evrard (1999) & $0.98( \pm 0.20)$ & $0.22( \pm 0.25)$ & $0.24( \pm 0.30)$ \\
& Novicki et al. (2002) $(z<0.3)$ & $0.54( \pm 0.31)$ & $-0.06( \pm 0.37)$ & $-0.02( \pm 0.43)$ \\
& Novicki et al. (2002) (0.3<z<0.6) & $0.10( \pm 0.62)$ & $-0.48( \pm 0.73)$ & $-0.34( \pm 0.83)$ \\
& & & & \\
$E_{z}^{-1} L-T$ & & & & \\
(Fig. 4) & Ettori et al. (2002) & $-1.04( \pm 0.32)$ & $-1.48( \pm 0.38)$ & $-1.46( \pm 0.44)$ \\
$E_{z} M_{\text {tot }}-T$ & Allen et al. (2001c) & $-0.16( \pm 0.23)$ & $-0.72( \pm 0.28)$ & $-0.78( \pm 0.32)$ \\
(Fig. 5) & Ettori et al. (2002) & $-0.12( \pm 0.39)$ & $-0.14( \pm 0.46)$ & $-0.06( \pm 0.52)$ \\
& Finoguenov et al. (2001) & $-0.26( \pm 0.17)$ & $-0.16( \pm 0.21)$ & $-0.12( \pm 0.26)$ \\
$E_{z} M_{\text {gas }}-T$ & Allen et al. (2001c) & $0.02( \pm 0.39)$ & $0.04( \pm 0.45)$ & $0.06( \pm 0.52)$ \\
(Fig. 6) & Ettori et al. (2002) & $-0.26( \pm 0.23)$ & $-0.54( \pm 0.27)$ & $-0.54( \pm 0.32)$ \\
$E_{z}^{-1} L-E_{z} M_{\text {tot }}$ & Mohr et al. (1999) & $-0.10( \pm 0.20)$ & $-0.42( \pm 0.24)$ & $-0.42( \pm 0.28)$ \\
(Fig. 7) & Ettori et al. (2002) & $-0.60( \pm 0.17)$ & $-1.10( \pm 0.20)$ & $-1.12( \pm 0.25)$ \\
\hline & Reiprich \& Böhringer (2002) & $0.18( \pm 0.20)$ & $-0.86( \pm 0.25)$ & $-0.78( \pm 0.32)$ \\
\hline
\end{tabular}

sample 24 clusters with weak or no cooling flows in their cores and X-ray measurements from GINGA, ASCA and Einstein, (iii) Novicki et al. (2002, Tables 4 and 5), that use a sample of 53 low $(z<0.3)$ and 32 intermediate $(0.3<z<0.6)$ redshift clusters observed with $A S C A$. Considering that the best-fit results obtained from these samples do not include the correction by the cosmological factor $E_{z}$, we consider here in the same way our measurements without this correction (Fig. 4, panel on the left). In this case, the $L-T$ relation should evolve like $E_{z} \approx(1+z)^{0.6 / 0.9}$ (for a " $\Omega_{\mathrm{m}}=1-\Omega_{\Lambda}=0.3$ " universe) and we should measure $B=0.6-0.9$, if self-similar predictions are correct.

Moreover, we compare the distribution of our data corrected by $E_{z}$ with the cosmology-corrected relations obtained from resolved temperature of (iv) 20 nearby $T>3 \mathrm{keV}$ objects observed with BeppoSAX (Ettori et al. 2002) and (v) six clusters with redshift between 0.10 and 0.46 observed with Chandra from Allen et al. (2001c). A predicted $E_{z}^{-1} L-T$ relation constant in time, and $B_{z}=0$, should be then observed.

When we compare the distribution of our data not corrected by $E_{z}$ with the local relations obtained from samples (i)-(iii) (Fig. 4, panel on the left), we measure $B$ between 0.1 and 0.6. In particular, when we use as local reference the result by Arnaud $\&$ Evrard (1999), $B$ becomes significantly positive $(0.98 \pm 0.20)$, but with a $\chi^{2}$ statistic $\left(\chi^{2}=51.6\right.$, d.o.f. $\left.=27\right)$ that is worst by a $\Delta \chi^{2} \gtrsim 30$ than the others measurements, mainly because of the relative small error on the slope $A$. These measurements of $B$ are slightly lower than, but still consistent with, the expected value between 0.6 and 0.9. The evidence that $B$ tends to be lower then the predicted value seems to suggest the presence of a slight negative evolution, with clusters at higher redshift having lower bolometric luminosities for given temperature. However, the small departure from $B=0$ in our $L-T$ relation not corrected by $E_{z}$ agrees with previous determinations obtained from Mushotzky \& Scharf (1997), Fairley et al. (2000) and Holden et al. (2002). In particular, Holden et al. analyze a subsample of the present dataset with only 12 objects, nine of those were exposed with Chandra. On the other hand, this value of $B$ is significantly lower than the result presented in Vikhlinin et al. (2002). These authors have assembled 22 clusters in the redshift range 0.39-1.26 which were available with sufficient long exposure to ensure a good temperature determination in the Chandra archive as of Spring 2002. Single temperatures (with column density and metallicity fixed to Galactic value and 0.3 times the solar abundance, respectively) were estimated collecting photons within 0.5-1 $h_{50}^{-1} \mathrm{Mpc}$. X-ray luminosities were measured within $2 h_{50}^{-1} \mathrm{Mpc}$. Both in the spectral and spatial analysis, the central regions with $r=100 h_{50}^{-1} \mathrm{kpc}$ were excised if a sharply peaked surface brightness was detected. Once compared with the local $L-T$ distribution in Markevitch (1998), where the same procedure in the determination of temperatures and luminosities has been adopted, Vikhlinin et al. claim a detection significant at $8 \sigma$ confidence level of a positive evolution $(B=1.5 \pm 0.2)$.

To check what causes this relevant difference, we reevaluate $B$ for the sample by Vikhlinin et al., using the results by Markevitch (1998) for the low- $z$ reference relation. 
We apply to this sample the same analysis procedure applied to our set of 28 clusters, which is based on minimizing the $\chi^{2}$ in Eq. (7) while propagating uncertainties both in normalization and slope. We obtain $B=1.1 \pm 0.3$, with a deviation from zero of only $3.5 \sigma$. This significance decreases to $2.2 \sigma$ ( $B=0.62 \pm 0.28, \chi^{2}=22.0$ with 27 d.o.f.) when our estimates of $L\left(<R_{500}\right)$ (see asterisks in Fig. 3) are considered for our sample of 28 objects. This value of $B$ is well in agreement with the predicted range of $0.6-0.9$ expected when a face value of luminosity not corrected by $E_{z}^{-1}$ is considered.

Furthermore, it is worth noticing that the procedure adopted here defines the radius, $R_{\Delta}$, at which all the physical properties of a system are recovered (see discussion in Sect. 2) as a function of its mass (temperature), redshift and given overdensity. As we show in Fig. 2, the value of $R_{\Delta}$ varies considerably in the redshift range $0.4-1.3$ with respect to a fixed metric radius. Therefore, systematic differences between quantities (such as X-ray luminosity) measured at $2 h_{50}^{-1} \mathrm{Mpc}$ and $R_{500}$ are expected and, in fact, are observed. If we extrapolate further our estimates of luminosity up to $R_{200}$, on average increase of 8 per cent in $L\left(<R_{200}\right)$ with respect to $L\left(<R_{500}\right)$ is measured and a more significant evolution $(B \approx 0.7 \pm 0.3)$ is observed.

Therefore, we conclude that the mismatch between our and Vikhlinin et al. (2002) results is due to, in decreasing order of relevance, (i) the different fitting procedure (that reduces the significance of the evolution by propagating properly the errors on the best-fit parameters of the local relation), (ii) being our sample larger at higher redshift (e.g., we have 16 galaxy clusters at $z>0.6$ and 4 at $z>1$, whereas Vikhlinin et al. have 9 and 2 objects, respectively), (iii) systematic differences in the definition of the reference radius and in the procedure to estimate the gas temperature and luminosity (see also discussion at the end of Sect. 2.1 and Fig. 3).

We consider now the cosmological corrected $L-T$ relation, that should not evolve in the self-similar scenario. We compare our observed luminosities, which were corrected by the $E_{z}$ factor, to the best-fit relations obtained from the samples (iv-v) discussed above. We measure $B_{z}=-1.04 \pm 0.32$ when our data are compared to the determination by Ettori et al. (2002) at median redshift of 0.05 , and $B_{z}=-0.16 \pm 0.23$ when comparing to the results by Allen et al. (2001c) for clusters at median redshift of 0.28 (Fig. 4, panel on the right). When we consider only the 16 objects with $z>0.6, B_{z}$ is measured more significantly negative: $B_{z}=-1.48 \pm 0.38$ and $-0.72 \pm 0.28$, respectively. We also note that the $\chi^{2}$ is definitely improved (values between 2 and 9 with 15 d.o.f.) also when the $B$ parameter is evaluated ( $B$ values in the range $-0.5 \pm 0.7$ and $0 \pm 0.3$ )

In agreement with a value $B$ lower than 0.6 , a $B_{z}<0$ indicates that a marginal detection of negative evolution (i.e. clusters at higher redshift have lower luminosities for a given $T$ ) appears when the relation is properly corrected by its cosmological dependence and compared to local estimates obtained under the same condition (i.e. all the photons are considered to recover both temperature and luminosity and the $E_{z}$ factor is taken into account as done in Allen et al. 2001c and Ettori et al. 2002).

\subsection{Evolution in the $M_{\mathrm{tot}}-T$ relation}

We compare our distribution in the $E_{z} M_{\text {tot }}-T$ plane with the local relations measured by (i) Ettori et al. (2002; BeppoSAX data), (ii) Finoguenov et al. (2001) for their subsample of 26 clusters with spatially resolved temperatures and emissionweighted values larger than $3 \mathrm{keV}$ as measured with ASCA (we have re-fitted their relation after rescaling the mass measurements in our cosmological model and correcting them by the corresponding factor $E_{z}$ ) and (iii) Allen et al. (2001c; Chandra data). In Fig. 5 we show our constraints on the evolution parameter $B_{z}$. Overall, there is no evidence of any evolution with values of $B_{z}$ always consistent with zero: $B_{z}=-0.12 \pm 0.39$ (Ettori et al. 2002), $B_{z}=-0.26 \pm 0.17$ (Finoguenov et al. 2001) and $B_{z}=0.02 \pm 0.39$ (Allen et al. 2001c). Also if we restrict the measurements to the subsample of clusters with $z>0.6$, we obtain $B_{z}$ consistent with zero: $B_{z}=-0.14 \pm 0.46$ (Ettori et al. 2002), $B_{z}=-0.16 \pm 0.21$ (Finoguenov et al. 2001) and $B_{z}=0.04 \pm 0.45$ (Allen et al. 2001c).

\subsection{Evolution in the $M_{\text {gas }}-T$ relation}

Figure 6 shows that there is no significant evolution in the $M_{\text {gas }}-T$ relation corrected by $E_{z}$ when it is compared with local estimates from Mohr et al. (1999) and Ettori et al. (2002). For this relation, we find $B_{z}=-0.10 \pm 0.20$ and $-0.26 \pm 0.23$ for these two reference analyses, thus corresponding to a detection of a negative evolution at $\lesssim 1 \sigma$ confidence level (in case of no evolution, $B_{z}=0$ ). A slightly more significant evolution is detected when $z>0.6$ clusters are used: $B_{z}=-0.42 \pm 0.24$ and $-0.54 \pm 0.27$, respectively. Still, it is worth noticing that, as observed in the $L-T$ relation, if any hint of evolution can be detected, this points toward a negative evolution, i.e. clusters tend to have lower X-ray luminosity and gas mass at higher redshift for given temperature. We underline that this is only a marginal $(<2 \sigma)$ evidence, even if confirmed from both measurements of $\mathrm{X}$-ray luminosity and gas mass.

\subsection{Evolution in the $L-M_{\text {tot }}$ relation}

The cosmologically corrected $L-M_{\text {tot }}$ relation (Fig. 7) appears consistent with a case of slightly negative evolution (i.e. $B_{z}<$ 0 ) that affects particularly the X-ray luminosities at redshift greater then 0.6: we measure $B_{z}=-0.60 \pm 0.17(-1.10 \pm 0.20$ for objects with $z>0.6)$ and $0.18 \pm 0.20(-0.86 \pm 0.25$ at $z>0.6$ ) when compared with the results in Ettori et al. (2002) and Reiprich \& Böhringer (2002), respectively. Note that we have obtained and considered the best-fit constraints on the $E_{z}^{-1} L_{\mathrm{bol}}-E_{z} M_{500}$ relation for the 85 objects (out of 106) in the Reiprich \& Böhringer (2002) sample with quoted X-ray temperature larger than $3 \mathrm{keV}$. Even though the scatter that we measure in this relation is the highest among the scaling laws investigated, the trend observed for systems with lower luminosities for given mass at higher redshifts agrees with the results presented on the $L-T$ relation. 


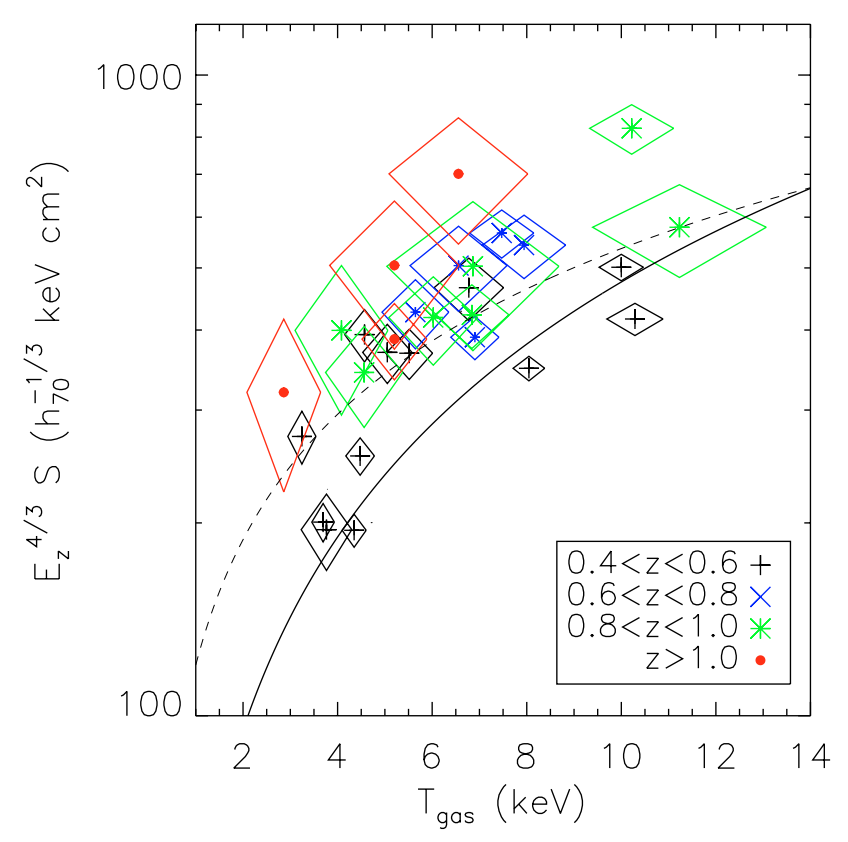

Fig. 9. Distribution of the entropy values measured at $0.1 \times R_{200}$ versus gas temperature. The distribution shows a Spearman's rank correlation significant at 99.9 per cent against the null-hypothesis that no-correlation is present. A rescaling factor of $E_{z}^{4 / 3}$ is used to compare these values to what is expected from hydrodynamical simulations, $S \approx 50\left(f_{\text {gas }} / 0.106\right)^{-2 / 3} T_{\mathrm{keV}} h_{70}^{-4 / 3} \mathrm{keV} \mathrm{cm}^{2}$ (solid line, from Ponman et al. 1999 and normalized to the median gas fraction measured in our sample; dashed line, $S \approx 120 T_{\mathrm{keV}}^{0.65}$, from Ponman et al. 2003).

\subsection{Evolution in the $S-T$ relation}

As a further diagnostic to trace the evolution of the ICM properties, we analyze the relation between entropy, $S$, and gas temperature. Starting from the work by Ponman et al. (1999), entropy has received a considerable attention as a diagnostic of the physical processes that establish the thermal status of the diffuse baryons in clusters (e.g., Tozzi \& Norman 2001; Voit et al. 2002). For the first time, we attempt here to trace the evolution of the ICM entropy, whose analysis has been limited so far to low- $z$ clusters. To this purpose, we measure the quantity $S=T_{\text {gas }} / n_{\mathrm{e}}^{2 / 3}$ at $0.1 \times R_{200}$ within each cluster as done by Ponman and collaborators for nearby systems. With the above definition, the quantity $S$ relates to the thermodynamical entropy $K$ of the system as $K \propto \log S$.

In Fig. 9, we plot the values of $S$ as a function of the gas temperature. The predicted scaling of $S \sim T$ is in agreement with the overall fit of the data $(S=(407 \pm 20) \times$

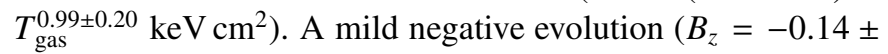
$0.04, \chi_{\min }^{2}=151$ with 27 d.o.f.) is observed when all our sample is compared with the local estimate of the "entropy ramp" by Ponman et al. (2003; see Fig. 9, panel on the left). However, the high $\chi^{2}$ value warns that this is not a good modelization of the data distribution, mainly for their large internal scatter. If we consider only the objects at $z>0.6,0.8$ and 1 , respectively, we observe consistently $B_{z} \approx 0.3$ with a deviation from no evolution $\left(B_{z}=0\right)$ at the $2-3 \sigma$ confidence level, with a reduced $\chi^{2}$ of about 1 (e.g., $B_{z}=0.26 \pm 0.06, \chi^{2} /$ d.o.f. $=18.5 / 15$ at $z>0.6, B_{z}=0.32 \pm 0.08, \chi^{2} /$ d.o.f. $=10.6 / 10$ at $\left.z>0.8\right)$.

\subsection{Discussion on the observed evolution}

As for the $E_{z}^{-1} L-T$ and $E_{z} M_{\text {gas }}-T$ relations, we detect a mild negative evolution $\left(B_{z} \sim(-0.1,-1.0)\right.$, with a deviation from the non-evolution case, $B_{z}=0$, by less than $3.2 \sigma$ ) that becomes more relevant when subsamples with higher cut in redshift are considered. For the 16 objects with $z>0.6$, we measure $B_{z}$ in the range $[-1.5,-0.4]$ with deviation from zero significant at $3.9 \sigma$ (see Table 4 ). This result points to a scenario in which clusters at higher redshift have in general lower $\mathrm{X}$-ray luminosities and gas masses than what predicted from the self-similar model. This trend appears more clearly when the entropy of these hot $\left(T_{\text {gas }}>3 \mathrm{keV}\right)$ systems, evaluated at $0.1 R_{200}$, is compared with local determinations. A mild positive evolution $\left(B_{z} \simeq 0.3\right)$ is then detected in the $E_{z}^{4 / 3} S-T$ relation, in particular when objects at redshift larger than 0.6 are considered.

The evolution of the $L-T$ and $M_{\mathrm{gas}}-T$ relations is a sensitive test for non-gravitational heating models (e.g. Tozzi \& Norman 2001; Bialek et al. 2001) and carries information about the source of this heating and the typical redshift at which it took place. The numerical simulations by Bialek et al. (2001) suggest that $M_{\text {gas }}$ is lower at higher redshift for fixed temperature, when the ICM is heated to an initial entropy of about $100 \mathrm{keV} \mathrm{cm}^{2}$, well before the cluster collapse takes place. This effect is more evident for smaller systems, thus making the $M_{\text {gas }}-T$ relation steeper at increasing redshift. A marginal, negative evolution in the $L-T$ relation is predicted by the semi-analytical model by Tozzi \& Norman (2001), when an epoch-independent entropy background of $K=0.3 \times 10^{34} \mathrm{ergs} \mathrm{cm}^{2} \mathrm{~g}^{-5 / 3}$ is combined with cooling in a $\Lambda C D M$ universe (see their Fig. 11 for emission-weighted temperature above $2 \mathrm{keV}$ ). We conclude that the observed negative evolution in the $L-T$ and $M_{\text {gas }}-T$ relations are in agreement with predictions from models that pre-heat the ICM with a nonevolving entropy floor at the level of about $100 \mathrm{keV} \mathrm{cm}^{2}$. In any case, this picture has been recently questioned by Ponman et al. (2003), who find no evidence for isentropic cores in groups.

A more controversial point is whether pre-heating can also account for the positive evolution of entropy in central cluster regions. If we are looking just at the effect of an entropy floor, which has been previously created by an extra-heating mechanism, the central regions of clusters would be characterized by an entropy level which does not change with time. Therefore, after applying the cosmological rescaling, the quantity $E_{z}^{4 / 3} S$ should be positively evolving. However, this evolution turns out to be stronger than that measured from the clusters at $z>0.6$, $(1+z)^{0.3}$, thus excluding that our measured evolution is the effect of a $z$-independent entropy floor. Furthermore, since most of our clusters are quite hot systems, they should sample the regime where entropy is dominated by gravitational shocks, rather than by a pre-collapse entropy floor. Indeed, in the standard scenario of pre-heating, one expects a minimum entropy level to be created in central regions of groups and poor clusters, while the ICM entropy structure should be left almost unaffected for hot systems, like those included in our sample. More in general, the emerging scenario from the entropy properties of low- and high $-z$ systems is calling for alternative 


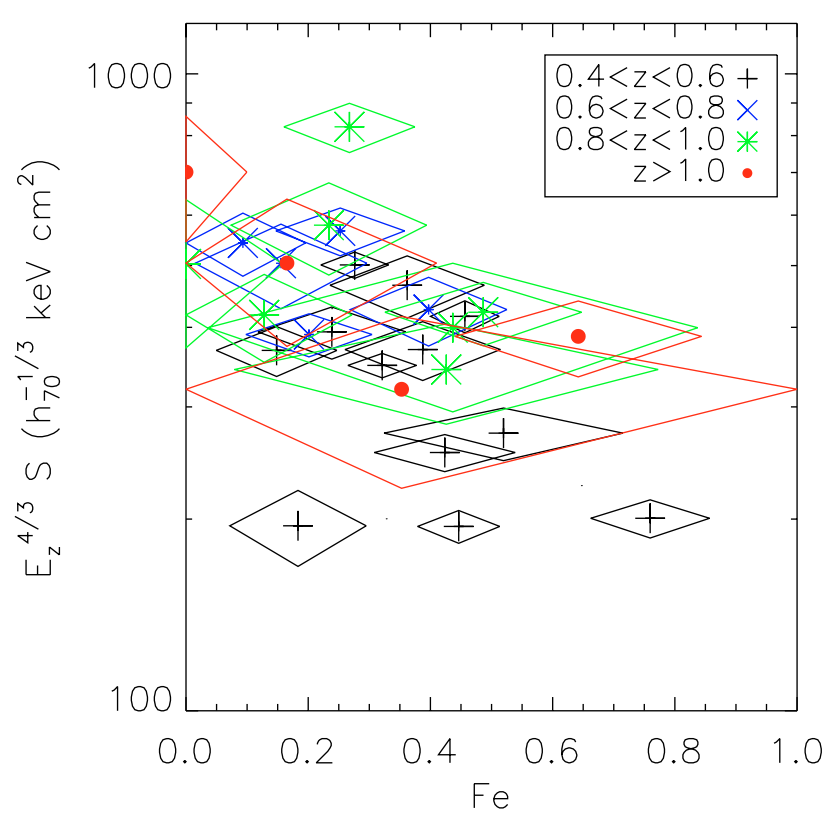

Fig. 10. Distribution of the entropy values measured at $0.1 \times R_{200}$ versus the Iron abundance measured with respect to the solar ratios in Anders \& Grevesse (1989). The Spearman's rank correlation is significant at 99.0 per cent against the no-correlation case.

interpretations, such as pre-heating with differential entropy amplification, as recently proposed by Voit et al. (2003), or a suitable combination of radiative cooling and heating by feedback energy release (e.g., Tornatore et al. 2003; Kay et al. 2003).

A further possibility is that we are looking at the combined effect of non-gravitational heating and radiative cooling. Although each one of these two mechanisms provides an excess entropy, hydrodynamical simulations of clusters have shown that their combined effect may induce non-trivial consequences in the entropy properties of the ICM (Tornatore et al. 2003). For instance, an episode of impulsive heating may cause the creation on an excess entropy, thus increasing the gas cooling time and suppressing cooling in central cluster regions. Subsequently, cooling starts acting again on the gas, gradually decreasing its entropy as gas flows toward the cluster center. In this case, one should observe a gradual reduction of the net entropy detected at the cluster center. Whether this is what we are actually observing requires a more sophistical numerical and semi-analytical modeling. In any case, there is little doubt that the evolution of gas entropy in central cluster regions keeps track of the past ICM thermal evolution. As such, its measurement provides unique information about the latest epoch at which a significant amount of energy has been injected into the ICM and of the involved energy budget.

As a diagnostic to connect the thermodynamics of the ICM to the past history of star formation, we have investigated the relation between entropy and the Iron content. (We remind that in these high $-z$ systems Iron is the only element that we can sample in their metal budget.) As shown in Fig. 10, we have a statistically significant indication that values of the central entropies are lower in correspondence to metal-richer clusters. This result, along with the evidence that systems at lower temperature stores lower amount of entropy at the same fraction of the virial radius, is an alternative way to show what we reported in a previous paper (Tozzi et al. 2003): clusters at lower temperature appear to have higher Iron abundance (see Baumgartner et al. 2003 for a discussion of this trend as observed in the ASCA galaxy cluster archive).

It is now well-established (e.g. Pettini 2003; Finoguenov et al. 2002) that most of the star formation and Iron production happened at $z \gtrsim 2$, i.e. before the final collapse of the accreting baryons into clusters, and preferentially in low-entropy structures like groups along cosmic filaments. Groups are the high$z$ precursor of later-forming rich clusters, which maintain their structure and survive for a few crossing times after they merge into the cluster environment (as suggested by the frequently observed cool cores in relaxed systems e.g. Vikhlinin et al. 2001 and corroborated by hydrodynamical simulations Motl et al. 2003; Tormen et al. 2003). This implies that the shorter the time elapsed from the aggregation of these clumps in a larger system, the larger the number of survival regions at low entropy and high metallicity. It is worth noticing that these stable subclusters should contribute significantly to all the X-ray emission-weighted observables because of their high gas density. Therefore, from an observational point of view, systems with higher metallicity should appear with lower gas temperature and be considered as dynamically younger. This supports what is shown by the surface brightness profiles, with centrally peaked emission, which is characteristic of relaxed systems, found to be less evident as more distant clusters are considered.

\section{Summary and conclusions}

We have studied the evolution of the scaling relations between observed and derived physical properties of the ICM and dark matter potential in a sample of $28 \mathrm{X}$-ray galaxy clusters with redshift in the range $z \simeq 0.4-1.3$ (median of 0.73 ). Using Chandra observations, we resolve their surface brightness on scales of a few arcsecs ( 1 arcsec corresponds to between 5 and $8 h_{70}^{-1} \mathrm{kpc}$ at these redshifts for the assumed cosmology), out to a significant fraction (about 0.7 , as median value) of $R_{500}$.

In general, the surface brightness is well fitted by a single $\beta$-model. A double $\beta$-model is statistically not required, suggesting that these distant clusters do not show any significant central brightness excess. This may be indicative that systems at high redshift appear as structures in formation and not completely relaxed. By collecting all the photons within $\sim 0.5 \times R_{500}$, we have measured a single emission-weighted temperature (median value of $6.0 \mathrm{keV}$ ). The combination of the spatial and spectral analysis allows us to recover the gas luminosity (observed in the range 1.0-116.7 $\times 10^{44} h_{70}^{-2} \mathrm{erg} \mathrm{s}^{-1}$ ), gas mass $\left(0.6-18.1 \times 10^{13} h_{70}^{-5 / 2} M_{\odot}\right)$ and total gravitating mass (1.4-21.3 $\left.\times 10^{14} h_{70}^{-1} M_{\odot}\right)$ out to $R_{500}$. These quantities are used to quantify the evolution of the X-ray scaling relations in hot $\left(T_{\text {gas }} \gtrsim 3 \mathrm{keV}\right)$ clusters.

The slopes of all the investigated correlations tend to be higher than what is predicted by self-similar models. The largest deviations from the self-similar scaling relations occur in the $L-T$ and $M_{\text {gas }}-T$ relations (Figs. 4-6). We notice that the values of the slope propagate through the 
relations in a self-consistent way: for example, the observed dependence $L \sim M_{\text {tot }}^{1.88 \pm 0.42}$ and $M_{\text {tot }} \sim T^{1.98 \pm 0.30}$ should imply $L \sim T^{3.7 \pm 1.0}$ that is well in accordance with the measured slope of $3.72 \pm 0.47$. This is in accordance with the observed properties of gas-dependent quantities, such as luminosity and gas mass (e.g. Edge \& Stewart 1991; Fabian et al. 1994; Mushotzky \& Scharf 1997; Allen \& Fabian 1998; Mohr et al. 1999; Novicki et al. 2001; Ettori et al. 2002), and suggests that simple gravitational collapse is not the only process that governs the heating of baryons in the potential well of clusters also in the redshift range $0.4-1.3$. On the other hand, we do not observe any significant dependence of the gas mass fraction upon the temperature (see Fig. 8), ruling out the simple explanation that the increase in the gas budget as function of the mass of the system is responsible for the steepening of the $L-T$ relation.

To explain the observed correlations, a raise in the central entropy (and a corresponding suppression of the X-ray emissivity) is required and can be achieved either by episodes of non-gravitational heating due to supernovae and AGN (e.g., Evrard \& Henry 1991; Cavaliere et al. 1999; Tozzi \& Norman 2001; Bialek et al. 2001; Brighenti \& Mathews 2001; Babul et al. 2002; Borgani et al. 2002), or by selective removal of low-entropy gas through cooling (e.g. Pearce et al. 2001; Voit \& Bryan 2001; Wu \& Xue 2002), possibly regulated by some mechanism supplying energy feedback (e.g. the semianalytical approach proposed by Voit et al. 2002 and the numerical simulations discussed by Muanwong et al. 2002; Tornatore et al. 2003; Kay et al. 2003).

When the slope of the $E_{z} M_{\mathrm{tot}}-T$ relation is fixed to 1.5 , its high $-z$ normalization of $(0.37 \pm 0.02) \times 10^{14} h_{70}^{-1} M_{\odot} \mathrm{keV}^{-3 / 2}$ is well in agreement with results from low $-z$ samples (e.g. Nevalainen et al. 2000; Allen et al. 2001c). This lack of evolution follows from the prediction of hydrostatic equilibrium within virialized halos. In turn, this is consistent with the expectation that the $E_{z} M_{\text {tot }}-T$ relation is the least sensitive to the thermal status of the gas and, therefore, not significantly affected by non-gravitational processes. Still, the $M_{\text {tot }}-T$ relation observed at low redshift (e.g., Horner et al. 1999; Nevalainen et al. 2000; Finoguenov et al. 2001; Ettori et al. 2002) is known to have a normalization lower by about 30 per cent than that obtained from hydrodynamical simulations (e.g. Mathiesen \& Evrard 2001; cf. also Muanwong et al. 2002; Borgani et al. in preparation). It will be interesting to show how our measured $E_{z} M_{\text {tot }}-T$ for distant clusters compare with predictions from hydrodynamical simulations.

As for the $E_{z}^{-1} L-T$ and $E_{z} M_{\mathrm{gas}}-T$ relations, we detect a mild negative evolution $\left(B_{z} \sim(-0.1,-1.0)\right.$, with a deviation from the non-evolution case $B_{z}=0$ by less than $\left.3.2 \sigma\right)$ that becomes more relevant when subsamples with higher cut in redshift are considered (see Table 4 ). For the 16 objects with $z>0.6$, we measure $B_{z}$ in the range $[-1.5,-0.4]$ with deviation from zero significant at $3.9 \sigma$. This result points to a scenario in which clusters at higher redshift have in general lower $\mathrm{X}$-ray luminosities and gas masses than that predicted from the self-similar model. This trend appears more clearly when the entropy of these hot $\left(T_{\text {gas }} \gtrsim 3 \mathrm{keV}\right)$ systems, evaluated at $0.1 R_{200}$, is compared with local determinations. A mild positive evolution $\left(B_{z} \simeq 0.3\right)$ is then detected in particular when objects at redshift larger than 0.6 are considered. The cut at $z \geq 0.6$ allows us to improve significantly the fit when a $\chi^{2}$ statistic is applied to evaluate the level of evolution. Even though a powerlaw modelization is probably simplistic to represent the properties of the ICM as a function of redshift, it provides generally a very good fit when clusters with $z<0.6$ are not included.

As we discuss in Sect. 4.6, these observed evolutions are broadly consistent with predictions from models that pre-heat the ICM with a non-evolving entropy floor at the level of about $100 \mathrm{keV} \mathrm{cm}^{2}$. However, these models are in conflict with recent observations of non-isentropic cores in galaxy groups (e.g. Ponman et al. 2003; Mushotzky et al. 2003). More detailed analyses of simulated structure formation are required to confirm quantitatively the degree of the expected evolution.

Acknowledgements. We thank the anonymous referee for a careful reading of the manuscripts and suggestions that have improved the presentation of our work. We are grateful to Thomas Reiprich for his quick reply to our emails.

\section{References}

Allen, S. W., \& Fabian, A. C. 1998, MNRAS, 297, L57

Allen, S. W., Taylor, G. B., Nulsen, P. E. J., et al. 2001a, MNRAS, 324,842

Allen, S. W., Ettori, S., \& Fabian, A. C. 2001b, MNRAS, 324, 877

Allen, S. W., Schmidt, R. W., \& Fabian, A. C. 2001c, MNRAS, 328, L37

Allen, S. W., Schmidt, R. W., \& Fabian, A. C. 2002, MNRAS, 335, 256

Anders, E., \& Grevesse, N. 1989, Geochim. Cosmochim. Acta, 53, 197

Arnaud, K. A. 1996, Astronomical Data Analysis Software and Systems V, ed. G. Jacoby, \& J. Barnes, ASP Conf. Ser., 101, 17

Arnaud, M., \& Evrard, A. E. 1999, MNRAS, 305, 631

Babul, A., Balogh, M. L., Lewis, G. F., \& Poole, G. B. 2002, MNRAS, 330, 329

Bevington, P. R., \& Robinson, D. K. 1992, Data reduction and error analysis for the physical sciences (McGraw-Hill)

Bialek, J. J., Evrard, A. E., \& Mohr, J. J. 2001, ApJ, 555, 597

Borgani, S., Rosati, P., Tozzi, P., et al. 2001, ApJ, 561, 13

Borgani, S., Governato, F., Wadsley, J., et al. 2002, MNRAS, 336, 409

Baumgartner, W. H., Loewenstein, M., Horner, D. J., \& Mushotzky, R. F. 2003, ApJ, in press [astro-ph/0309166]

Brighenti, F., \& Mathews, W. G. 2001, ApJ, 553, 103

Bryan, G. L., \& Norman, M. L. 1998, ApJ, 495, 80

Cagnoni, I., Elvis, M., Kim, D.-W., et al. 2001, ApJ, 560, 86

Cavaliere, A., \& Fusco-Femiano, R. 1976, A\&A, 49, 137

Cavaliere, A., Menci, N., \& Tozzi, P. 1999, MNRAS, 308, 599

David, L. P., Slyz, A., Jones, C., et al. 1993, ApJ, 412, 479

De Grandi, S., \& Molendi, S. 2002, ApJ, 567, 163

Dickey, J. M., \& Lockman, F. J. 1990, ARA\&A, 28, 215

Donahue, M., Gaskin, J. A., Patel, S. K., et al. 2003, ApJ, 598, 190

Edge, A. C., \& Stewart, G. C. 1991, MNRAS, 252, 414

Ettori, S., \& Fabian, A. C. 1999, MNRAS, 305, 834

Ettori, S. 2000, MNRAS, 318, 1041

Ettori, S., De Grandi, S., \& Molendi, S. 2002, A\&A, 391, 841

Ettori, S., Tozzi, P., \& Rosati, P. 2003, A\&A, 398, 879

Evrard, A. E., \& Henry, J. P. 1991, ApJ, 383, 95

Evrard, A. E., Metzler, C. A., \& Navarro, J. F. 1996, ApJ, 469, 494 
Fabian, A. C., Crawford, C. S., Edge, A. C., \& Mushotzky, R. F. 1994, MNRAS, 267, 779

Fairley, B. W., Jones, L. R., Scharf, C., et al. 2000, MNRAS, 315, 669

Finoguenov, A., Reiprich, T. H., \& Böhringer, H. 2001, A\&A, 368, 749

Finoguenov, A., Jones, C., \& Böhringer Ponman, T. J. 2002, ApJ, 578, 74

Gioia, I. M., Maccacaro, T., Schild, R. E., et al. 1990, ApJS, 72, 567

Gioia, I. M., Henry, J. P., Mullis, C. R., Ebeling, H., \& Wolter, A. 1999, AJ, 117, 2608

Henry, J. P., \& Henriksen, M. J. 1986, ApJ, 301, 689

Henry, J. P., Gioia, I. M., Mullis, C. R., et al. 2001, ApJ, 553, L109

Holden, B. P., Stanford, S. A., Squires, G. K., et al. 2002, AJ, 124, 33

Jeltema, T. E., Canizares, C. C., Bautz, M. W., et al. 2001, ApJ, 562, 124

Kaiser, N. 1986, MNRAS, 222, 323

Kaiser, N. 1991, ApJ, 383, 104

Kay, S. T., Thomas, P. A., \& Theuns, T. 2003, MNRAS, 343, 608

Markevitch, M. 1998, ApJ, 504, 27

Markevitch, M., \& Vikhlinin, A. 2001, ApJ, 563, 95

Marshall, H. L., Tennant, A., Grant, C. E., et al. 2003, Proc. SPIE, 5165 [astro-ph/0308332]

Mathiesen, B. F., \& Evrard, A. E. 2001, ApJ, 546, 100

Maughan, B. J., Jones, L. R., Ebeling, H., et al. 2003, ApJ, 587, 589

McCarthy, I. G., Babul, A., \& Balogh, M. L. 2002, ApJ, 573, 515

Mohr, J. J., Mathiesen, B., \& Evrard, A. E. 1999, ApJ, 517, 627

Motl, P. M., Burns, J. O., Loken, C., Norman, M. L., \& Bryan, G. 2003, ApJ, in press [astro-ph/0302427]

Muanwong, O., Thomas, P. A., Kay, S. T., \& Pearce, F. R. 2002, MNRAS, 336, 527

Mullis, C. R., Mc Namara, B. R., Quintana, H., et al. 2003, ApJ, 594, 154

Mushotzky, R. F. 1984, Phys. Scr., T7, 157

Mushotzky, R. F., Scharf, C. A. 1997, ApJ, 482, L13

Mushotzky, R. F., Figueroa-Feliciano, E., Loewenstein, M., \& Snowden, S. L. 2003 [astro-ph/0302267]

Navarro, J. F., Frenk, C. S., \& White, S. D. M. 1997, ApJ, 490, 493
Nevalainen, J., Markevitch, M., \& Forman, W. 2000, ApJ, 536, 73

Nousek, J. A., \& Shue, D. R. 1989, ApJ, 342, 1207

Novicki, M. C., Sornig, M., \& Henry, J. P. 2002, AJ, 124, 2413

Pearce, F. R., Jenkins, A., Frenk, C. S., et al. 2001, MNRAS, 326, 649

Perlman, E. S., Horner, D. J., Jones, L. R., et al. 2002, ApJS, 140, 265

Pettini, M. 2003 [astro-ph/0303272]

Ponman, T. J., Cannon, D. B., \& Navarro, J. F. 1999, Nature, 397, 135

Ponman, T. J., Sanderson, A. J. R., \& Finoguenov, A. 2003, MNRAS, 343, 331

Pratt, G. W., \& Arnaud, M. 2002, A\&A, 394, 375

Reiprich, T. H., \& Böhringer, H. 2002, ApJ, 567, 716

Rosati, P., della Ceca, R., Norman, C., \& Giacconi, R. 1998, ApJ, 492, L21

Rosati, P., Borgani, S., \& Norman, C. 2002, ARA\&A, 40, 539

Rosati, P., et al. 2003, AJ, in press [astro-ph/0309546]

Schindler, S., Guzzo, L., Ebeling, H., et al. 1995, A\&A, 299, L9

Spergel, D. N., Verde, L., Peiris, H. V., et al. 2003, ApJS, 148, 175

Stanford, S. A., Holden, B. P., Rosati, P., et al. 2001, ApJ, 552, 504

Thomas, P. A., Muanwong, O., Pearce, F. R., et al. 2001, MNRAS, 324,450

Tormen, G., Moscardini, L., \& Yoshida, N. 2003, MNRAS, submitted [astro-ph/0304375]

Tornatore, L., Borgani, S., Springel, V., et al. 2003, MNRAS, 342, 1025

Tozzi, P., \& Norman, C. 2001, ApJ, 546, 63

Tozzi, P., Rosati, P., Ettori, S., et al. 2003, ApJ, 593, 705

Vikhlinin, A., Mc Namara, B. R., Forman, W., et al. 1998, ApJ, 502, 558

Vikhlinin, A., Markevitch, M., \& Murray, S. S. 2001, ApJ, 549, L47

Vikhlinin, A., van Speybroeck, L., Markevitch, M., Forman, W. R., \& Grego, L. 2002, ApJ, 578, L107

Voit, G. M., Bryan, G. L., Balogh, M. L., \& Bower, R. G. 2002, ApJ, 576, 601

Weisskopf, M. C., Tanabaum, H. D., van Spebroeck, L. P., O’ Dell, S. L. 2000, Proc SPIE, 4012, in press [astro-ph/0004127]

White, S. D. M., Navarro, J. F., Evrard, A. E., \& Frenk, C. S. 1993, Nature, 366, 429 


\section{Online Material}


S. Ettori et al.: Scaling laws in high- $z$ X-ray galaxy clusters, Online Material $p 2$
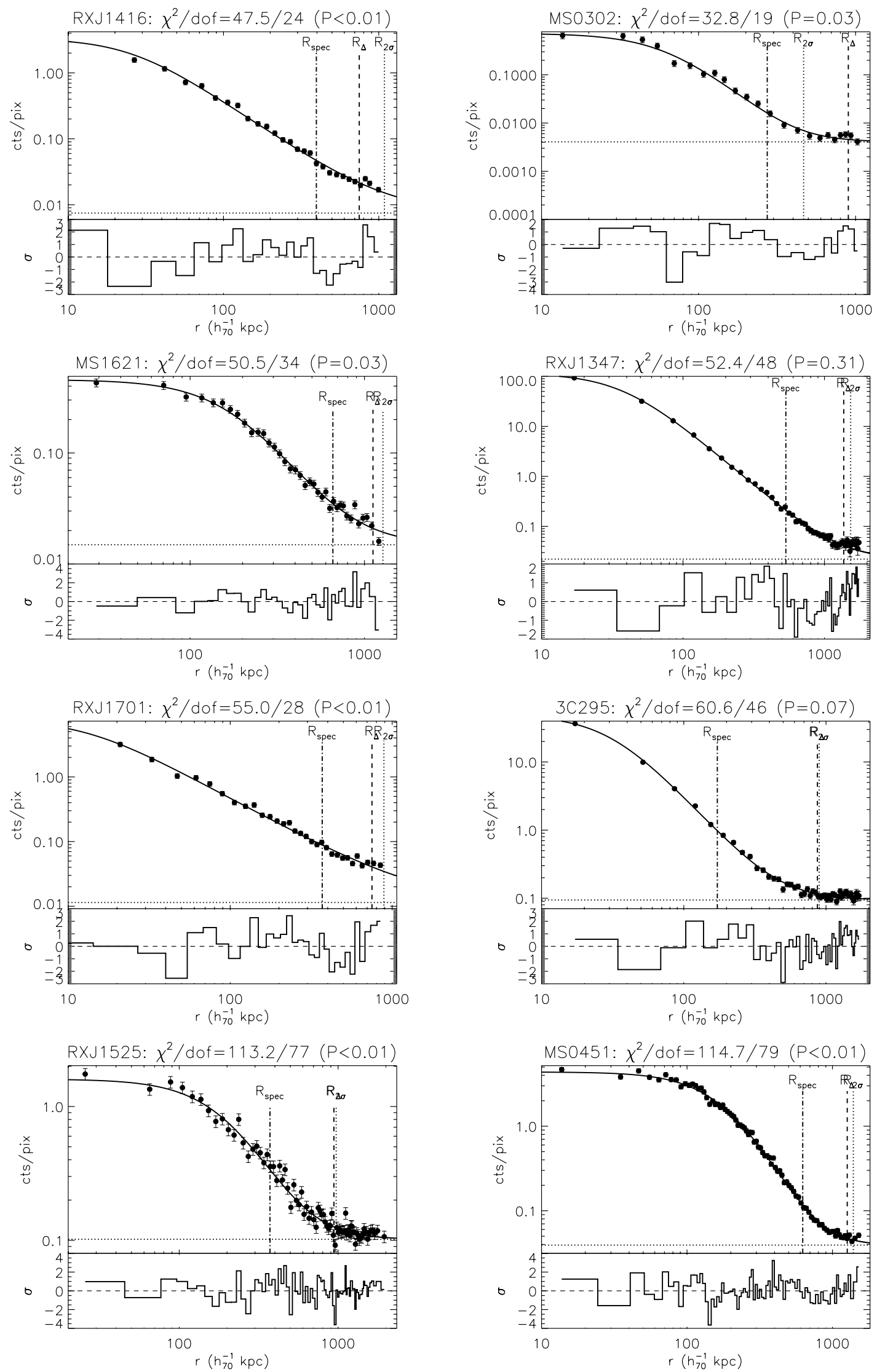

Fig. A.1. Data, best-fit $\beta$-model and residuals of the surface brightness profile of the high redshift galaxy clusters in our sample. Dotted lines indicate the best-fit background value. The location of $R_{\text {spec }}, R_{\Delta}$ and $R_{2 \sigma}$ is shown. 
S. Ettori et al.: Scaling laws in high $-z$ X-ray galaxy clusters, Online Material $p 3$
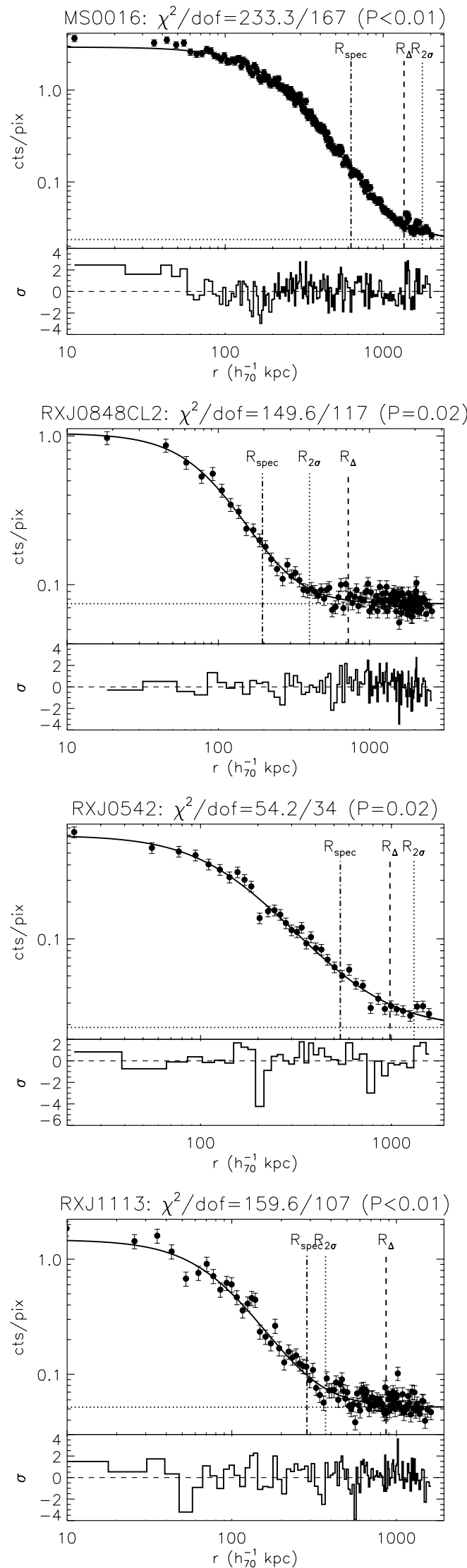
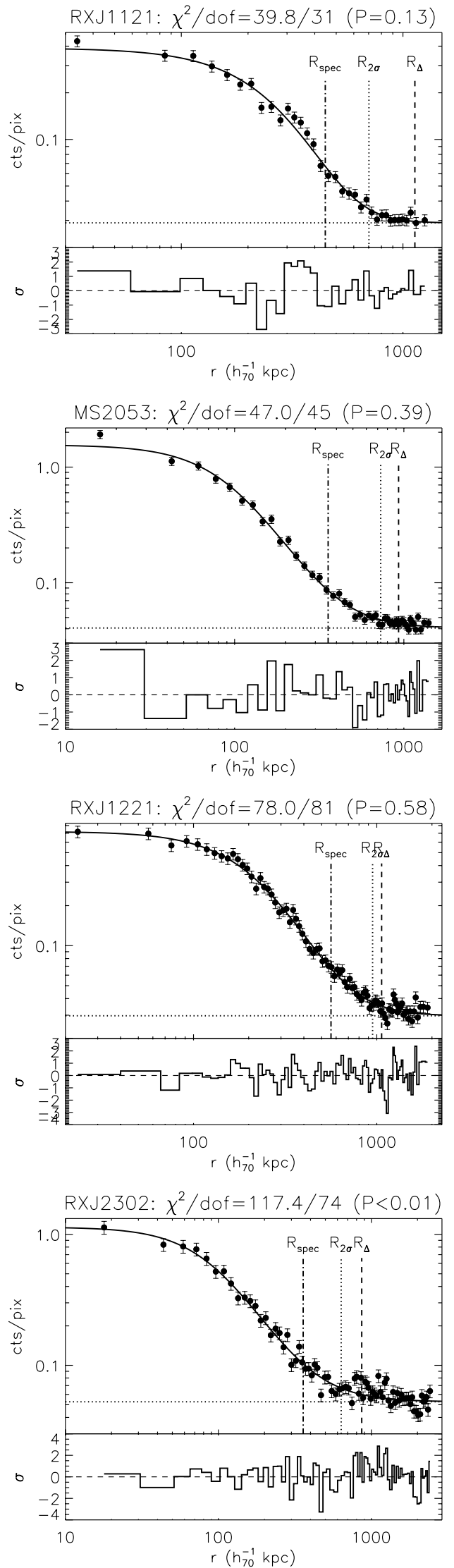

Fig. A.1. continued. 
S. Ettori et al.: Scaling laws in high-z X-ray galaxy clusters, Online Material p 4
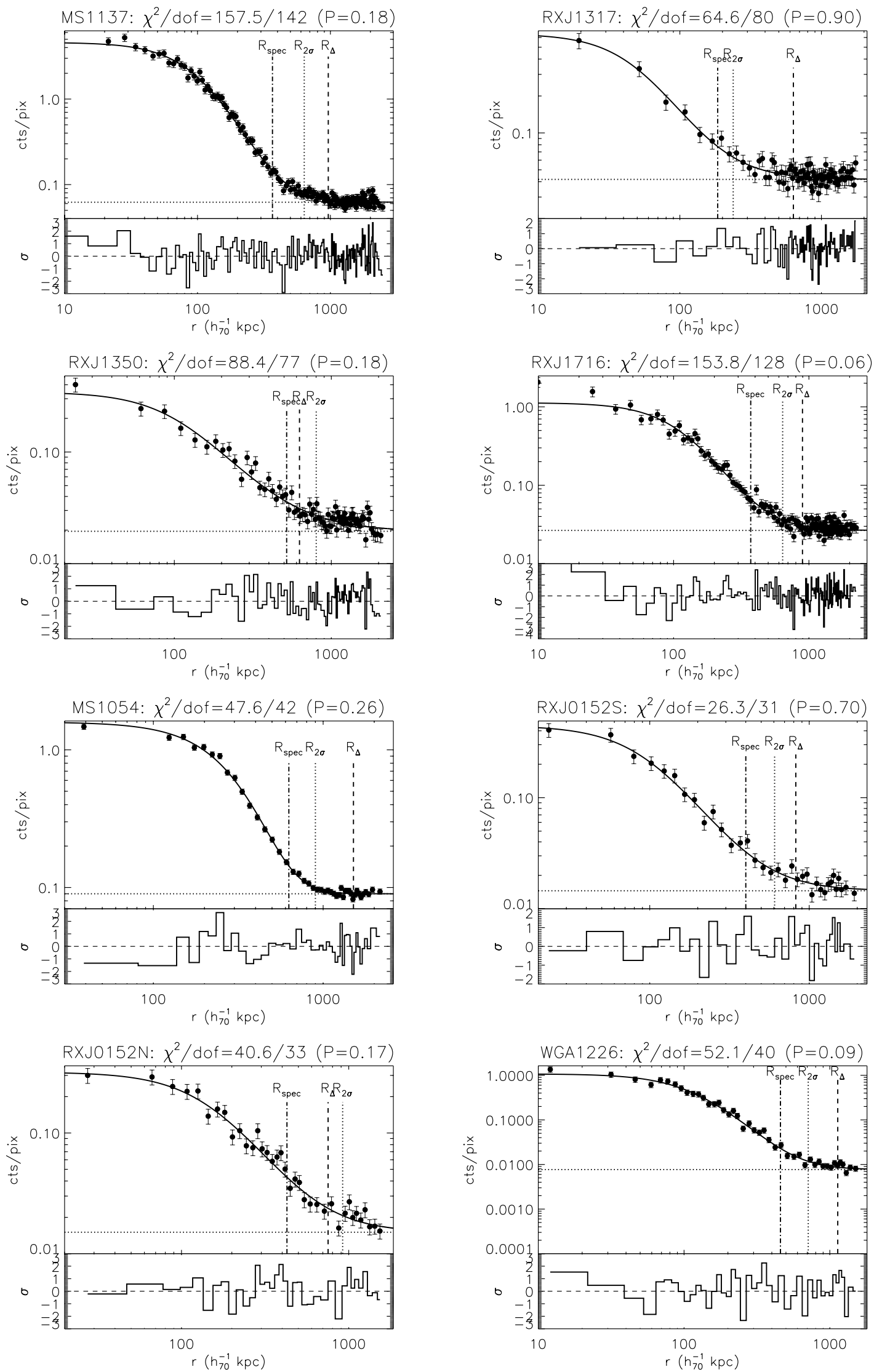

Fig. A.1. continued. 
S. Ettori et al.: Scaling laws in high $-z$ X-ray galaxy clusters, Online Material p 5
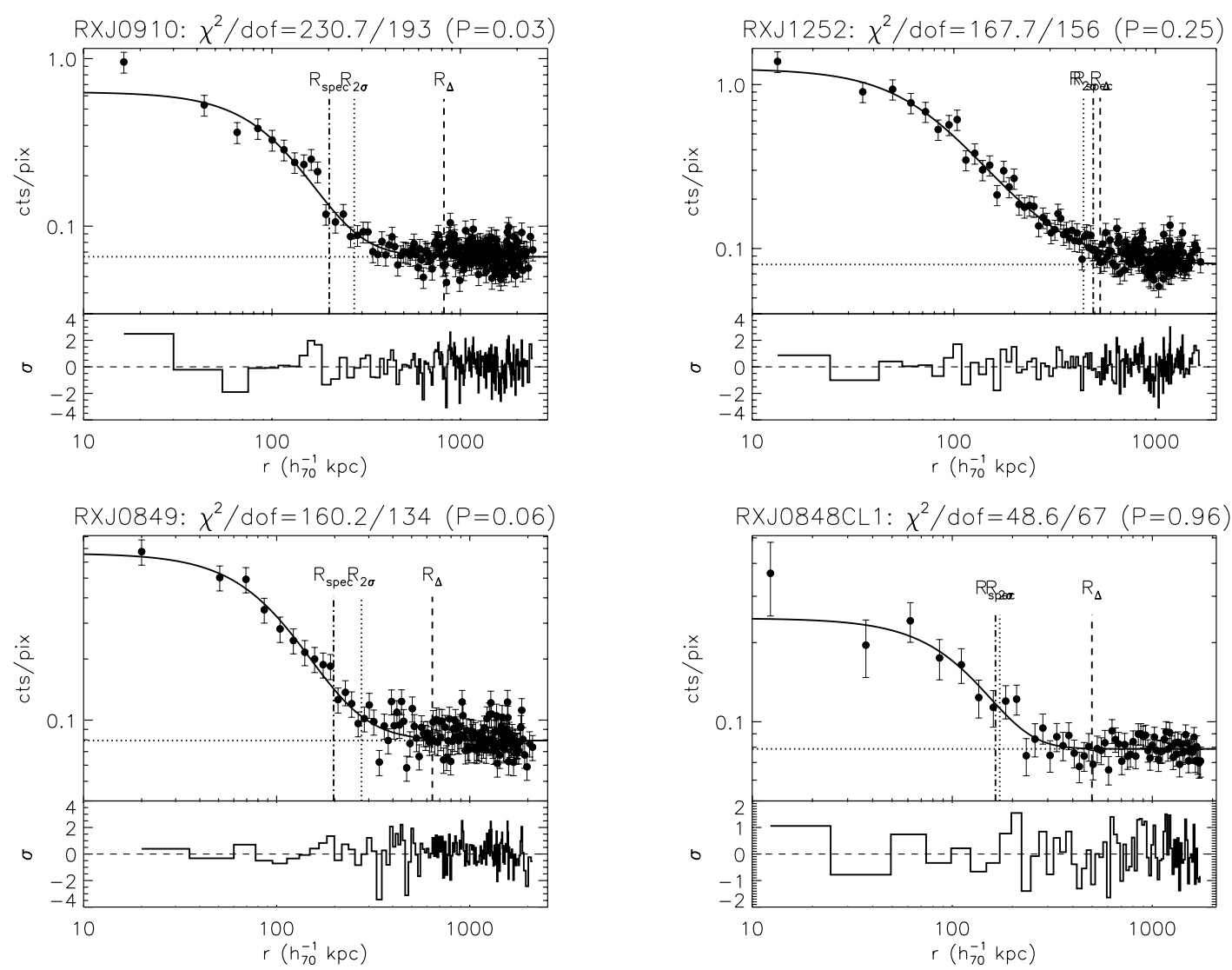

Fig. A.1. continued. 